\title{
Baryogenesis and gravity waves from a UV-completed electroweak phase transition
}

\author{
Benoit Laurent $\odot^{*}$ and James M. Cline $\odot^{\dagger}$ \\ McGill University, Department of Physics, 3600 University St., Montréal, Quebec, H3A2T8 Canada \\ Avi Friedlander $\odot^{\ddagger}$ \\ Queen's University, Department of Physics \& Engineering Physics Astronomy Kingston, \\ Ontario, K7L 3N6 Kingston, Canada \\ Dong-Ming $\mathrm{He} \odot^{\S}$ \\ University of Science and Technology of China, Hefei, Anhui 230026 and Universiteit van Amsterdam, \\ Science Park 904, Amsterdam 1098XH, Netherlands
}

Kimmo Kainulainen $\oplus^{\|}$

Department of Physics, P.O.Box 35 (YFL), FIN-40014 University of Jyväskylä, Finland and Helsinki Institute of Physics, P.O. Box 64, FIN-00014 University of Helsinki, Finland

\author{
David Tucker-Smith \\ Department of Physics, Williams College, Williamstown, Massachusetts 01267, USA
}

(Received 8 March 2021; accepted 11 May 2021; published 10 June 2021)

\begin{abstract}
We study gravity wave production and baryogenesis at the electroweak phase transition in a real singlet scalar extension of the Standard Model, including vectorlike top partners, to generate the $C P$ violation needed for electroweak baryogenesis (EWBG). The singlet makes the phase transition strongly first order through its coupling to the Higgs boson, and it spontaneously breaks $C P$ invariance through a dimension-five contribution to the top quark mass term, generated by integrating out the heavy top quark partners. We improve on previous studies by incorporating updated transport equations, compatible with large bubble wall velocities. The wall speed and thickness are computed directly from the microphysical parameters rather than treating them as free parameters, allowing for a first-principles computation of the baryon asymmetry. The size of the $C P$-violating dimension-five operator needed for EWBG is constrained by collider, electroweak precision, and renormalization group running constraints. We identify regions of parameter space that can produce the observed baryon asymmetry or observable gravitational wave (GW) signals. Contrary to standard lore, we find that for strong deflagrations, the efficiencies of large baryon asymmetry production and strong GW signals can be positively correlated. However, we find the overall likelihood of observably large GW signals to be smaller than estimated in previous studies. In particular, only detonation-type transitions are predicted to produce observably large gravitational waves.
\end{abstract}

DOI: $10.1103 /$ PhysRevD.103.123529

\section{INTRODUCTION}

*benoit.laurent@mail.mcgill.ca

†avi.friedlander@queensu.ca

*dong-ming.he@student.uva.nl

\$cline@physics.mcgill.ca

kimmo.kainulainen@jyu.fi

`dtuckers@williams.edu

Published by the American Physical Society under the terms of the Creative Commons Attribution 4.0 International license. Further distribution of this work must maintain attribution to the author(s) and the published article's title, journal citation, and DOI. Funded by SCOAP ${ }^{3}$.
Phase transitions in the early Universe provide an opportunity for probing physics at high scales through cosmological observables, in particular, if the transition is first order. In that case, it may be possible to explain the origin of baryonic matter through electroweak baryogenesis (EWBG) [1-4] or variants thereof [5]. Such transitions can also produce relic gravitational waves (GWs) that may be detectable by future experiments like LISA [6,7], BBO [8], DECIGO [9,10] and AEDGE [11].

It is remarkable that even though the electroweak phase transition (EWPT) is a smooth crossover in the Standard 
Model (SM) [12,13], it can become first order with the addition of modest new physics input, in particular, a singlet scalar coupling to the Higgs [14-23] that can also be probed in collider experiments [24-35]. There have been many studies of such new physics models with respect to their potential to produce observable cosmological signals [36-51]. However, it is challenging to make a firstprinciples connection between microphysical models and the baryon asymmetry or GW production since these can be sensitive to the velocity $v_{w}$ and thickness $L_{w}$ of the bubble walls in the phase transition, which are numerically demanding to compute [52-63]. Most previous studies that encompass EWBG and GW studies of the EWPT, therefore, leave $v_{w}$ and $L_{w}$ as free parameters. This limitation was addressed recently in Ref. [64], which undertook a comprehensive investigation of the EWPT enhanced by coupling the Higgs boson to a scalar singlet with $Z_{2}$ symmetry. The simplicity of this model facilitates doing an exhaustive search of its parameter space.

In the present work we continue the investigation started in Ref. [64], which determined $v_{w}$ and $L_{w}$ over much of the model parameter space, but did not try to predict the baryon asymmetry or GW production. Moreover, that study was limited to subsonic wall speeds, due to a breakdown of the fluid equations that determine the friction on the wall. Recently, a set of improved fluid equations was postulated in Refs. [65,66] that do not suffer from the subsonic limitation. We use these in the present work in order to fully explore the parameter space, where high $v_{w}$ can be favorable to observable GWs, and also compatible with EWBG. It will be shown that for strong deflagrations the fluid velocity in front of the wall saturates and even decreases with increasing wall velocity $v_{w}$. Since the walls become thinner at the same time, the baryon asymmetry is enhanced at larger wall velocities for these transitions, becoming positively correlated with a strong GW signal. Despite this positive correlation, we find that producing the observed baryon asymmetry together with a GW signal detectable in next generation observations is not possible, in contrast to previous estimates $[34,44]$. The difference comes from several factors working in the same direction. For example, we find larger wall velocities and thicknesses than Ref. [44], which suppress the baryon asymmetry. Moreover, our GW fits include a recently derived suppression factor due to shock reheating $[67,68]$, which leads to a much weaker $\mathrm{GW}$ signal for strong deflagrations.

A further improvement in this work is to present an ultraviolet completion of the effective coupling that gives rise to the $C P$ violation needed for EWBG. We introduce heavy vectorlike top partners, which when integrated out induce a $C P$-violating coupling of the singlet scalar $s$ to top quarks, giving the source term for EWBG. ${ }^{1}$ Although the

\footnotetext{
${ }^{1}$ Hints of the presence of such a particle in LHC data were recently presented in Ref. [69].
}

effective operator description of this term is quite adequate for quantitatively understanding EWBG [70,71], its resolution in terms of underlying physics is necessary for quantifying how large its coefficient can be, consistent with laboratory constraints. We present the details in Sec. II, including comprehensive collider limits on the top partners and the subsequent constraints on the effective theory. The finite-temperature effective potential of the theory is also outlined there, along with a discussion of cosmological constraints on the small explicit breaking of the $Z_{2}$ symmetry that is necessary for EWBG.

The paper continues in Sec. III with a brief description of our methodology for finding the high-temperature firstorder phase transitions and characterizing their strength. This is followed in Sec. IV by a detailed account of how the bubble wall speed and shape are determined. The techniques for computing the baryon asymmetry and GW production are described in Sec. V. We present the results of a Monte Carlo exploration of the model parameter space with respect to these observables in Sec. VI, with emphasis on the interplay between successful EWBG and potentially observable GWs. Conclusions are given in Sec. VII, followed by several Appendixes containing details about construction of the finite-temperature effective potential, solving junction conditions for the phase transition boundaries, and predicting GW production.

\section{II. $Z_{2}$-SYMMETRIC SINGLET MODEL}

We study the $Z_{2}$-symmetric singlet scalar extension of the SM with a real singlet $s$ coupled to the Higgs doublet $H$. The scalar potential is

$$
\begin{aligned}
V(H, s)= & \mu_{h}^{2} H^{\dagger} H+\lambda_{h}\left(H^{\dagger} H\right)^{2}+\frac{\lambda_{h s}}{2}\left(H^{\dagger} H\right) s^{2} \\
& +\frac{\mu_{s}^{2}}{2} s^{2}+\frac{\lambda_{s}}{4} s^{4} .
\end{aligned}
$$

We work in unitary gauge, which consists of taking $H=h / \sqrt{2}$; the Goldstone bosons still contribute to the one-loop and thermal corrections, but they are set to zero in the tree-level potential. We assume $\mu_{h}^{2}<0$ and $\mu_{s}^{2}<0$, which implies that the potential has nontrivial minimums at $v \equiv h= \pm\left|\mu_{h}\right| / \sqrt{\lambda_{h}} \approx 246 \mathrm{GeV}, s=0$ and $h=0$, and $s= \pm\left|\mu_{s}\right| / \sqrt{\lambda_{s}}$. The scalar fields' mass in the vacuum can then be written in terms of the parameters of the potential as $m_{h}^{2}=-2 \mu_{h}^{2} \approx(125 \mathrm{GeV})^{2}$ and $m_{s}^{2}=$ $-\lambda_{h s} \mu_{h}^{2} /\left(2 \lambda_{h}\right)+\mu_{s}^{2}$.

The other relevant interaction of $s$ is a dimension-five operator yielding an imaginary contribution to the top quark mass [72]:

$$
\mathcal{L}_{\mathrm{BG}}=-\frac{y_{t}}{\sqrt{2}} h \bar{t}_{L}\left(1+i \frac{s}{\Lambda}\right) t_{R}+\text { H.c. }
$$


This term will be ignored during the discussion on the phase transition; however, it is essential for generating the baryon asymmetry since it gives the $C P$-violating source term when $s$ temporarily gets a vacuum expectation value (VEV) in the bubble walls of the electroweak phase transition. In Eq. (2) we have adopted a special limit of a more general model, in which the dimension-five contribution is purely imaginary. This can be understood as a consequence of imposing $C P$ in the effective Lagrangian, with $s$ coupling like a pseudoscalar, $s \rightarrow-s$. Hence it is consistent to omit terms odd in $s$ in the scalar potential (1), even though Eq. (2) is odd in $s$. The $C P$ symmetry prevents a VEV from being generated for $s$ by loops.

The effective operator is generated by integrating out a heavy singlet vectorlike top quark partner $T$, whose mass term and couplings to the third generation quarks $q_{L}=\left(t_{L}, b_{L}\right)$, Higgs, and singlet fields are

$y_{t} \bar{q}_{L} H t_{R}+\eta_{1} \bar{q}_{L} H T_{R}+i \eta_{2} \bar{T}_{L} s t_{R}+M \bar{T}_{L} T_{R}+$ H.c.

including also the SM $q_{L}$-Higgs coupling. This is invariant under $C P$ if $s \rightarrow-s .^{2}$ Integrating out $T$ leads to the effective operator in (2) with scale

$$
\Lambda=\frac{y_{t} M}{\eta_{1} \eta_{2}}
$$

We consider experimental constraints on the scale $\Lambda$ below.

In previous literature, thermal corrections were frequently approximated by including just the first term of the high-temperature expansion of the thermal functions presented in Appendix B. However, this approximation fails at temperatures below the mass of particles strongly coupled to the Higgs, as can happen in models with a high degree of supercooling. Therefore, we employ the full oneloop thermal functions. This will be shown to have a large impact on the values of the tunneling action, and thus of the nucleation temperature. In addition to the tree-level potential and the thermal corrections, we also include the oneloop correction and the thermal mass Parwani resummation [73]. The complete effective potential then becomes

$$
V_{\text {eff }}=V_{\text {tree }}+V_{\mathrm{CW}}+V_{T}+\delta V .
$$

We checked the dependence of our results on the thermal resummation method by redoing partial scans by using the alternative ring resummation method [74-76]. We found only minor differences, with the ring method allowing slightly more models with larger $m_{s}$, but not enough to have any effect on our final conclusions. More discussion and precise definitions of the effective potential components are given in Appendix A.

\footnotetext{
${ }^{2}$ The interaction term $i \eta_{3} \bar{T}_{L} s T_{R}$ also respects $C P$ for real $\eta_{3}$. We neglect it to simplify our analysis.
}

\section{A. Laboratory constraints}

It is important to determine how low the scale $\Lambda$ of the dimension-five operator in Eq. (4) can be since it has a strong impact on the baryon asymmetry $\eta_{b}$; in the limit of large $\Lambda, \eta_{b}$ scales as $1 / \Lambda$. The relevant masses and couplings are constrained by direct searches for the top partner and precision electroweak studies. Moreover, the properties of the singlet $s$ are constrained by collider searches.

After electroweak symmetry breaking, a Dirac mass term $\left(\bar{t}_{L}, \bar{T}_{L}\right)\left(\begin{array}{cc}m_{t} & \mu \\ 0 & M\end{array}\right)\left(\begin{array}{c}t_{R} \\ T_{R}\end{array}\right)$ is generated for $t, T$, with $m_{t}=y_{t} v / \sqrt{2}$ and $\mu=\eta_{1} v / \sqrt{2}$ that is diagonalized by separate rotations on $\left(t_{R}, T_{R}\right)$ and $\left(t_{L}, T_{L}\right)$, with mixing angles

$\tan 2 \theta_{L}=2 \frac{M \mu}{M^{2}-m_{t}^{2}-\mu^{2}}, \quad \tan 2 \theta_{R}=2 \frac{m_{t} \mu}{M^{2}+\mu^{2}-m_{t}^{2}}$.

For example, we consider a benchmark point with $\eta_{1}=$ 0.55 and a physical $T$ mass $M_{T}=800 \mathrm{GeV}$, which correspond to $M=794 \mathrm{GeV}$ and mixing angles $\theta_{L}=$ 0.126 and $\theta_{R}=0.027$. The relations between $y_{t}$ and the physical top mass differ from the SM ones by less than $1 \%$, which is allowed by current LHC constraints [77,78]. For sufficiently large $\eta_{2}$, decays of $T$ to $h t / Z t / W b$ induced by mixing are highly subdominant to $T \rightarrow s t$, and searches for vectorlike top partners that focus on the former channels are evaded. Near the Goldstone-equivalent limit (which should apply reasonably well for $M_{T}=800 \mathrm{GeV}$ and relatively small $s$ masses, $m_{s} \sim 100 \mathrm{GeV}$ ), the branching ratio for $T \rightarrow s t$ is

$$
B(T \rightarrow s t) \simeq \frac{\eta_{2}^{2}}{\eta_{2}^{2}+2 \eta_{1}^{2}}
$$

We roughly estimate from Refs. [79,80] that for $M_{T}=800 \mathrm{GeV}$, vectorlike quark searches that target SM final states are evaded provided $B(T \rightarrow s t) \gtrsim 90 \%$, corresponding to $\eta \gtrsim 2.4$ for our benchmark point. Reference [81] (see Fig. 1 of contribution 5; also [82]) has reinterpreted collider bounds to constrain the parameter space $\left(m_{s}, M_{T}\right)$ for models in which $T \rightarrow s t$ dominates, finding that top partner masses above $\sim 750 \mathrm{GeV}$ are allowed in the case where $s$ decays $100 \%$ into two gluons. This is true in our model, where the dominant $s$ decays are induced by the loop diagrams shown in Fig. 1. One can estimate that the gluon final state dominates over that of $b$
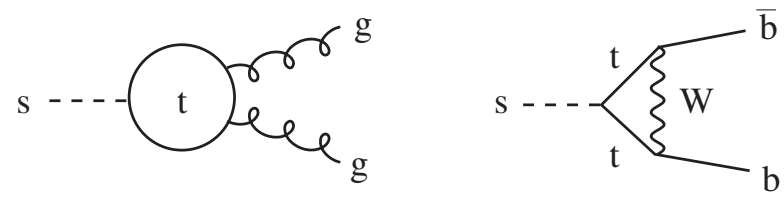

FIG. 1. Feynman diagrams for decay of the singlet $s$. The decay into gluons is by far the dominant channel. 

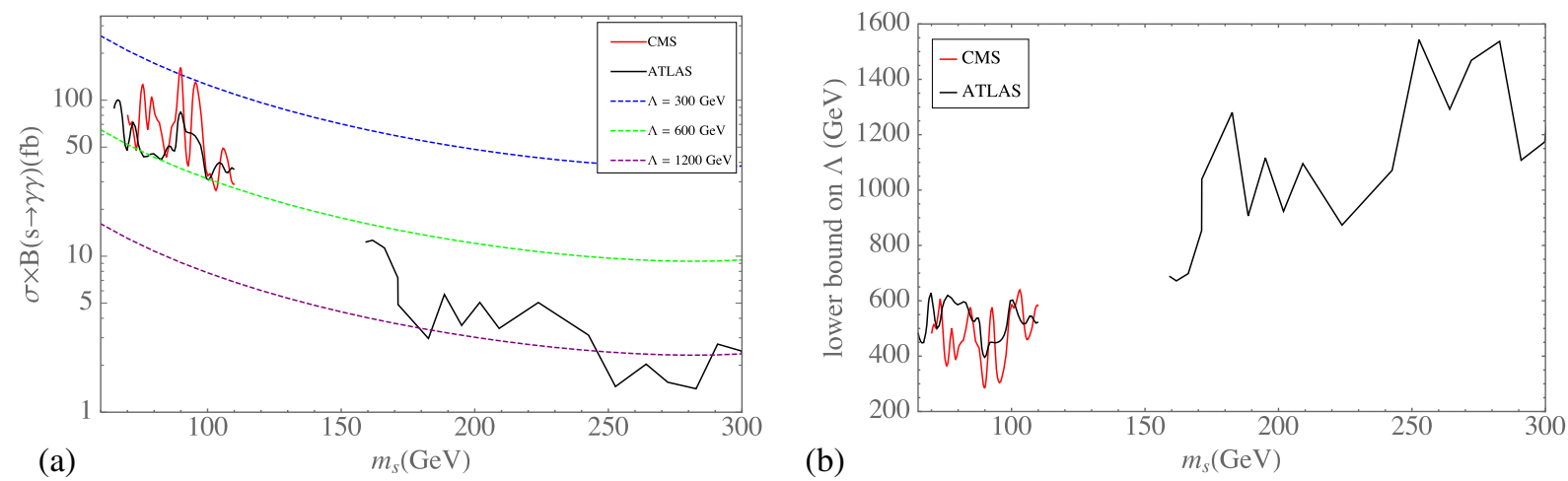

FIG. 2. Left (a): Experimental limits from ATLAS [86,87] and CMS [88] for resonant production of $s$ by $g g$ fusion followed by decays into photons (solid lines) versus predictions at different values of $\Lambda$. Right (b): Corresponding lower bounds on $\Lambda$.

quarks by a factor of $\left(g_{s}^{2} m_{s} / g_{w}^{2} m_{b}\right)^{2} \gtrsim 10^{3}$ and over decays into photons by $\left(g_{s} / e\right)^{4} \sim 300$. Precision electroweak data constrain the additional contributions to the oblique parameters, especially $T$, which is corrected by [83]

$\Delta T=T_{\mathrm{sm}} s_{L}^{2}\left(-\left(1+c_{L}^{2}\right)+s_{L}^{2} r+2 c_{L}^{2} \frac{r}{r-1} \ln r\right) \lesssim 0.1$,

where $T_{\mathrm{sm}}=1.19$ is the $\mathrm{SM}$ value, $c_{L}=\cos \theta_{L}$, $s_{L}=\sin \theta_{L}$, and $r=\left(M_{T} / m_{t}\right)^{2}$; the upper limit is from Sec. X of [84]. The benchmark point chosen above almost saturates this constraint, giving $\Delta T \simeq 0.09$.

There are also direct searches for resonant production of the singlet by gluon-gluon fusion. The coupling of $s$ to $t$ in the mass eigenstate basis is $y_{s t}=\eta_{2} \cos \theta_{R} \sin \theta_{L} \sim \eta_{2} \theta_{L}$, while that to $T$ is $y_{s T}=-\eta_{2} \cos \theta_{L} \sin \theta_{R} \sim-\eta_{2} \theta_{R}$. The squared matrix element for the decays $s \rightarrow g g$ is [85]

$$
|\mathcal{M}|^{2}=\left(\frac{\alpha_{s}}{\pi}\right)^{2} m_{s}^{4}\left|\sum_{i=t, T} \frac{y_{s i}}{m_{i}} \tau_{i}\left[\sin ^{-1}\left(\tau_{i}^{-1 / 2}\right)\right]^{2}\right|^{2},
$$

where $\tau_{i}=4 m_{i}^{2} / m_{s}^{2}$. The parton-level production cross section for $g g \rightarrow s$ is $\hat{\sigma}=\pi|\mathcal{M}|^{2} \delta\left(\hat{s}-m_{s}^{2}\right) /(256 \hat{s})$, where the 256 comes from averaging over gluon colors and spins. Integrating this over the gluon parton distribution functions gives the hadron-level cross section

$$
\begin{aligned}
\sigma(p p \rightarrow s) & =\frac{\pi}{256 m_{s}^{4}}|\mathcal{M}|^{2} \mathcal{L}_{g} \\
& \equiv \frac{\pi}{256 m_{s}^{4}}|\mathcal{M}|^{2} \int_{m_{s}^{2} / s}^{1} \frac{d x}{x}\left[x f_{g}\right](x)\left[x f_{g}\right]\left(m_{s}^{2} / s x\right),
\end{aligned}
$$

in which dependence on $m_{s}$ drops out except in the parton luminosity factor $\mathcal{L}_{g}$. This production is probed via decays $s \rightarrow \gamma \gamma$, whose branching ratio is approximately $B(s \rightarrow \gamma \gamma)=(8 / 9) \alpha^{2} / \alpha_{s}^{2}$ [85]. For the dominant $s \rightarrow g g$ decay into gluons, in principle, LHC dijet resonance searches could be constraining, but these exist only for $m_{s} \gtrsim 500 \mathrm{GeV}$, which is beyond the range of interest for the present study. To a good approximation, $\sigma(p p \rightarrow s)$ is determined by $m_{s}$ and $\Lambda$. In Fig. 2(a) we show limits from ATLAS [86,87] and CMS [88] on $\sigma B(s \rightarrow \gamma \gamma)$ as a function of $m_{s}$, along with the predictions for various $\Lambda$, and in Fig. 2(b) we show the associated lower bounds on $\Lambda$. In the low-mass region $\left(65 \mathrm{GeV}<m_{s}<110 \mathrm{GeV}\right)$, lower bounds on $\Lambda$ range roughly from $400 \mathrm{GeV}$ to $650 \mathrm{GeV}$; in the intermediate-mass region $\left(110 \mathrm{GeV}<m_{s}<160 \mathrm{GeV}\right), \Lambda$ is not yet constrained by diphoton resonance searches, and for much of the high-mass region $\left(m_{s}>160 \mathrm{GeV}\right), \Lambda$ is bounded to be above $1 \mathrm{TeV}$. For our subsequent scans of parameter space, we adopt a fixed reference value for $\Lambda$,

$$
\Lambda_{\text {ref }}=540 \mathrm{GeV} \text {, }
$$

which is large enough to be consistent with much of the low- $m_{s}$ region. Because $\Lambda_{\text {ref }}$ is well below the lower bounds on $\Lambda$ in the high-mass region, we confine our scans to $m_{s}<160 \mathrm{GeV}$ for consistency. ${ }^{3}$

The constraints from precision electroweak data, diphoton resonance searches, and vectorlike quark searches are shown in the $\eta_{1}-\eta_{2}$ plane in Fig. 3 for $M_{T}=800 \mathrm{GeV}$, where we approximate the $T$ search constraints by the requirement $B(T \rightarrow s t)>0.9$, and for $M_{T}=1300 \mathrm{GeV}$, heavy enough to evade $T$ searches for any $B(T \rightarrow s t)$. For the chosen $m_{s}$, it is apparent that the reference value $\Lambda=$ $540 \mathrm{GeV}$ is attainable for $\eta_{2} \gtrsim 2.5$ for $M_{T}=800 \mathrm{GeV}$ and $\eta_{2} \gtrsim 3$ for $M_{T}=1300 \mathrm{GeV}$. For slightly heavier $s$ in the window $110 \mathrm{GeV}<m_{s}<160 \mathrm{GeV}$, diphoton resonance searches are evaded and the red contours disappear. In this case even lower values of $\Lambda$ are allowed provided one is willing to consider larger values of $\eta_{2}$. Since the baryon

\footnotetext{
${ }^{3}$ Although we do not pursue this point here, lower values of $\Lambda$ are consistent with $m_{s}>160 \mathrm{GeV}$ if $B(s \rightarrow \gamma \gamma)$ is suppressed, for example, by a dominant invisible decay channel; LHC constraints on $t \bar{t}$ plus missing energy $[89,90]$ are in that case evaded for $M_{T} \gtrsim 1350 \mathrm{GeV}$.
} 

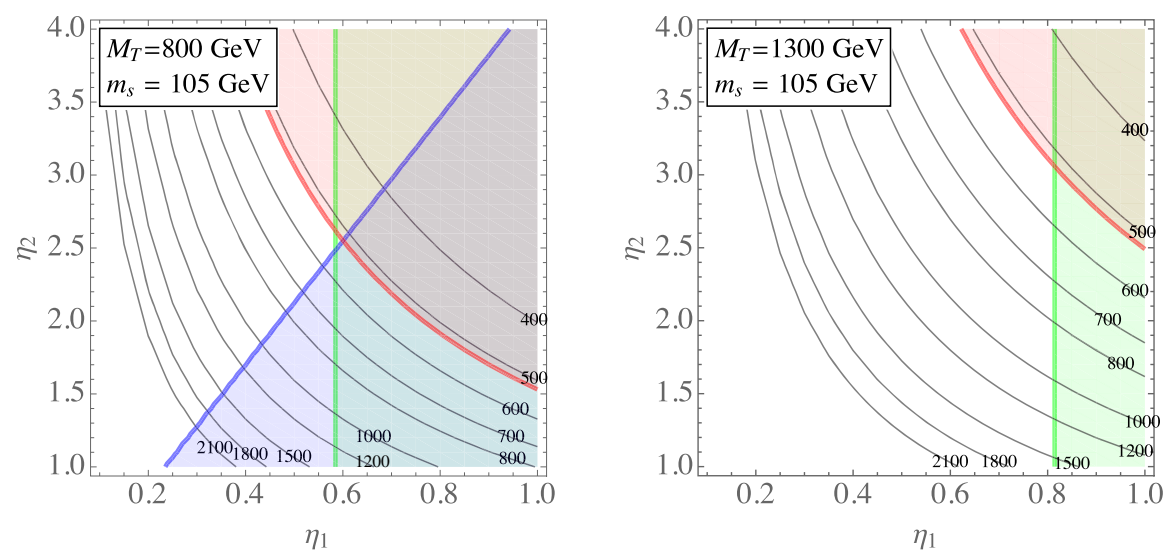

FIG. 3. For selected $T$ and $s$ masses, constraints on $\eta_{1}$ and $\eta_{2}$ from precision electroweak data (green), diphoton resonance searches $[87,88]$ (red), and searches for vectorlike quarks [79] (blue), along with contours of $\Lambda$ in GeV. The allowed region is unshaded.

asymmetry $\eta_{b}$ scales roughly as $1 / \Lambda$, it is straightforward to reinterpret our final results for larger (or smaller) $\Lambda$. From the results of Sec. VI, one can infer that a significant fraction of models remain viable for baryogenesis for $\Lambda=$ $2 \Lambda_{\text {ref }}$ (or for even larger $\Lambda$ ), a scale consistent with more modest couplings, $\eta_{2} \sim 1.5$.

Allowing for very large values of $\eta_{2}$ could invalidate the effective theory above the heavy top partner threshold $M$ at scales only slightly larger than $M$, which would require us to specify additional new physics in order to have a complete description. There are two principal challenges arising from the running of the couplings,

$$
\begin{gathered}
\frac{d \eta_{2}}{d \ln \mu} \cong \frac{\eta_{2}^{3}}{4 \pi^{2}} \\
\frac{d \lambda_{s}}{d \ln \mu} \cong \frac{9 \lambda_{s}^{2}}{8 \pi^{2}}-\frac{3 \eta_{2}^{4}}{2 \pi^{2}}+\frac{\lambda_{s} \eta_{2}^{2}}{2 \pi^{2}},
\end{gathered}
$$

where $\mu$ denotes the renormalization scale. The most serious problem is that for large values of $\eta_{2}$, the selfcoupling $\lambda_{s}$ is quickly driven to zero, and the scalar potential becomes unstable. The second is that $\eta_{2}$ reaches a Landau pole at somewhat higher scales. The first problem could be ameliorated by coupling additional scalars to $s$, without impacting our results for EWBG or GWs. For this reason, we do not limit the scope of our investigation based on the running of $\lambda_{s}$. Regarding the second problem, we note that even for $\eta_{2}=3$, the Landau pole is nearly an order of magnitude above $M$, which we consider to be an acceptably large range of validity for the effective theory.

\section{B. Explicit breaking of $Z_{2}$ symmetry}

Since we are considering a scenario where the $Z_{2}$ symmetry $s \rightarrow-s$ is spontaneously broken during the early Universe and restored at the EWPT, domain walls form before the EWPT, and the Universe will consist of domains with random signs of the $s$ condensate. The source term for EWBG that arises from Eq. (2) is linear in $s$, resulting in baryon asymmetries of opposite signs that could average to zero after completion of the EWPT. To avoid this outcome, the $Z_{2}$ symmetry should be explicitly broken by potential terms

$$
V_{b}=\mu_{b} s\left(h^{2}-v^{2}\right)+\mu_{b}^{\prime} s^{3}
$$

with small coefficients $\mu_{b}, \mu_{b}^{\prime}$. We have used the freedom of shifting $s$ by a constant to remove a possible tadpole of $s$ at the true vacuum $(h, s)=(v, 0)$.

The presence of the biasing potential $V_{b}$ can prevent the baryon washout in several ways. First, if the transition to the broken- $s$ phase is of second order, even a small tilt can suffice to make the lower-energy vacuum dominate. Second, in a first-order transition, symmetry breaking terms can bias the bubble nucleation rates to prefer the lower-energy vacuum. Indeed, the number of bubbles nucleated during the transition is $n \sim \int_{t_{c}}^{t_{*}} \mathrm{~d} t \Gamma(t)$, where $t_{*}$ is the time when transition completes, and $\Gamma(t) \sim \exp \left(-S_{3} / T\right)$. Writing the action as $S_{3 \pm}=\bar{S}_{3} \mp \delta S$ in the two respective vacua, the relative number density of bubbles in each phase at the end of the transition becomes $n_{+} / n_{-} \approx \exp \left(2 \delta S_{*} / T_{*}\right)$. In general [91], $S_{3} \propto E$, where $E$ is the coefficient of the cubic term in the potential. Using this scaling, we may write $\delta S_{*}=\left(\delta E / E_{0}\right) \bar{S}_{3}^{*}$, where typically $S_{3}^{*} / T_{*} \approx 100$. In our model $E_{0} \approx\left(3 \lambda_{s}\right)^{3 / 2} T / 12 \pi$, so taking $V_{b}=\mu_{b^{\prime}} s^{3}$, corresponding to $\delta E=\mu_{b^{\prime}}$, and $T_{*} \approx 100 \mathrm{GeV}$, the condition for single-phase vacuum dominance becomes $\mu_{b^{\prime}} \gtrsim 0.1 \lambda_{s}^{3 / 2} \mathrm{GeV}$. Barring very large $\lambda_{s}$, this condition is easily met with no limitations on our analysis.

Even if a domain wall network forms, the higher-energy domains will collapse due to pressure gradients, and we should ensure that this process completes before the EWPT. The collapse starts with the acceleration of a wall at relative position $R$ according to $\ddot{R}=-\Delta V / \tau$, where $\tau \sim \sqrt{\lambda_{s}} w^{3}$ is the surface tension (distinct from the tension $\sigma$ used above in the nucleation estimate), $\Delta V \sim V_{b}(0, w) \sim \mu_{b}^{\prime} w^{3}$ is the 
difference in the vacuum energies, and $w \sim \mu_{s} / \sqrt{\lambda_{s}}$ is the singlet VEV. Using $H=1 / 2 t$ and $T \approx 100 \mathrm{GeV}$, one finds that walls reach light speed in time

$$
\frac{\delta t}{t}=\frac{\tau H}{\delta V} \sim 10^{-5} \sqrt{\lambda_{s}}\left(\frac{\mathrm{eV}}{\mu_{b^{\prime}}}\right),
$$

which is practically instantaneous on the timescales of interest, for reasonable values of $\mu_{b^{\prime}}$. We note that global symmetries like $Z_{2}$ are expected to be broken by quantum gravity effects, so that it could be reasonable to anticipate $\mu_{b}^{\prime} \sim v^{2} / M_{p} \sim 0.1 \mathrm{eV}$, which is large enough from the perspective of Eq. (15).

The higher energy domains subsequently collapse at the speed of light since there is no appreciable friction. The time required for this process to complete is determined by $R_{*}=2 a\left(t_{1}\right) \int_{t_{1}}^{t_{2}} d t / a(t)$, where $R_{*}$ is the comoving size of the domain wall separation. By the Kibble mechanism one expects that $R_{*}=A H_{*}^{-1}$ with $A \lesssim 1$, leading to the ratio of domain wall collapse to formation times $t_{2} / t_{1}=(1+A / 2)^{2}$. The temperature interval corresponding to this time interval is $\Delta T / T \approx A$, assuming that the growth phase also proceeded at the speed of light.

The temperature of the first phase transition $T_{1}$ can be estimated as that when $\partial^{2} V / \partial s^{2}$ becomes negative. In the approximation of neglecting $V_{b}$, and keeping only leading terms in the high- $T$ expansion, one finds $T_{1}^{2}-T_{c}^{2} \sim \lambda_{h} w_{c}^{2} / c_{s}$, where $T_{c}$ is the critical temperature of the EWPT, and $c_{s}=\left(3 \lambda_{s}+2 \lambda_{h s}\right) / 12$. Thus, the temperature difference between transitions is of order $\Delta T_{1 c} \sim \lambda_{h} w^{2} /\left(c_{s} T_{c}\right)$. Requiring that $\Delta T_{1 c} / T_{c}>A$ then gives

$$
A<\frac{12 \lambda_{h}}{3 \lambda_{s}+2 \lambda_{h s}} \frac{w_{c}^{2}}{T_{c}^{2}} \sim O(1) .
$$

Given that $A \sim\left(T_{*} / S_{3}^{*}\right)(\Delta T / T)_{*} \sim 10^{-2}-10^{-4}$ [52], this is a very weak constraint. We conclude that it is easy to avoid cosmological problems associated with the domain walls by small symmetry breaking terms that do not affect the rest of our analysis.

\section{PHASE TRANSITION AND BUBBLE NUCLEATION}

In the examples of interest for this work, the phase transition in the $Z_{2}$-symmetric singlet model proceeds in two steps: starting from the high-temperature global minimum $h=s=0$, a transition first occurs to nonzero $s$, while the Higgs field remains at $h=0$. This is followed by the EWPT, in which $s$ returns to zero and $h$ develops its VEV. The $h^{2} s^{2}$ interaction provides the potential barrier to make this a first-order transition.

As usual, the first-order transition occurs at the bubble nucleation temperature $T_{n}$, which is below the critical temperature $T_{c}$, where the two potential minima become degenerate,

$$
\left.V_{\text {eff }}\left(h, s, T_{c}\right)\right|_{\substack{h=0, s=w_{c}}}=\left.V_{\text {eff }}\left(h, s, T_{c}\right)\right|_{\substack{h=v_{c}, . \\ s=0}}
$$

Bubble nucleation occurs when the vacuum decay rate per unit volume $\Gamma_{d}$ becomes comparable to $H^{4}$, the Hubble rate per Hubble volume. The decay rate is [92]

$$
\Gamma_{d} \cong T^{4}\left(\frac{S_{3}}{2 \pi T}\right)^{3 / 2} \exp \left(-\frac{S_{3}}{T}\right)
$$

where $S_{3}$ is the $\mathrm{O}(3)$ symmetric action,

$$
S_{3}=4 \pi \int r^{2} d r\left(\frac{1}{2}\left(\frac{d h}{d r}\right)^{2}+\frac{1}{2}\left(\frac{d s}{d r}\right)^{2}+V_{\text {eff }}\right) .
$$

The precise criterion that we use for nucleation is

$$
\exp \left(-S_{3} / T_{n}\right)=\frac{3}{4 \pi}\left(\frac{H\left(T_{n}\right)}{T_{n}}\right)^{4}\left(\frac{2 \pi T_{n}}{S_{3}}\right)^{3 / 2},
$$

which is satisfied when $S_{3} / T_{n} \cong 140$ [93]. We used the package CosmoTransitions [94] to calculate $S_{3}$. The action obtained with the full potential can differ significantly from the commonly used thin wall approximation $[95,96]$ or the approximation of evaluating it along the minimal integration path for the potential [44]. We compare the predictions for nucleation of these approximations to the full one-loop result, for several exemplary models, in Table I. The approximate methods tend to underestimate the action,

TABLE I. Examples of the dimensionless tunneling action $S_{3} / T$, evaluated at $T=100 \mathrm{GeV}$, and ensuing nucleation temperatures, computed within the thin wall and minimal potential path (MPP) approximations, compared with the value obtained using the resummed one-loop potential. In the example, $\lambda_{s}=1$ and $\Lambda=540 \mathrm{GeV}$.

\begin{tabular}{lcccccrrr}
\hline \hline & & \multicolumn{3}{c}{$S_{3} /\left.T\right|_{T=100 \mathrm{GeV}}$} & & \multicolumn{3}{c}{$T_{n}(\mathrm{GeV})$} \\
\cline { 3 - 5 } \cline { 7 - 9 }$\lambda_{h s}$ & $m_{s}(\mathrm{GeV})$ & Thin wall & MPP & 1-loop & & Thin wall & MPP & 1-loop \\
\hline 1 & 120 & 234 & 277 & 427 & & 93.5 & 92.6 & 89.8 \\
1.7 & 200 & 68.7 & 101 & 151 & & 115.6 & 109.8 & 100.1 \\
3.2 & 300 & 37.9 & 36.8 & 54.3 & & 134.3 & 133.8 & 121.6 \\
\hline \hline
\end{tabular}


giving a higher nucleation temperature; hence we use the values derived from the full one-loop action in the following.

There are two complementary parameters for characterizing the strength of the first-order transition. One is the ratio of the Higgs VEV to the temperature at the time of nucleation $v_{n} / T_{n}$, which is especially relevant for EWBG, as we will discuss in Sec. V B. The other, which is more important for GW production, is the ratio of released vacuum energy density to the radiation energy density [97,98]:

$$
\alpha=\frac{1}{\rho_{\gamma}}\left(\Delta V-\frac{T_{n}}{4} \Delta \frac{d V}{d T}\right)
$$

where $\rho_{\gamma}=g_{*} \pi^{2} T_{n}^{4} / 30, g_{*}$ is the effective number of degrees of freedom in the plasma (we use $g_{*}=106.75$ ), and $\Delta$ denotes the difference between the unbroken and broken phase. Note that $\alpha$ quantifies the amount of supercooling that occurs prior to nucleation, which determines how much free energy is available for the production of GWs.

\section{WALL VELOCITY AND SHAPE}

The derivation of the wall velocity and field profiles is a technically demanding problem [52], that was first addressed in the context of Higgs plus singlet models in Refs. [56,58,99], in various approximations. One must solve the equations of motion (EOMs) for the scalar sector coupled to a perfect fluid,

$$
\begin{aligned}
E_{h}(z) \equiv & -h^{\prime \prime}(z)+\frac{d V_{\mathrm{eff}}\left(h, s ; T_{+}\right)}{d h} \\
& +\sum_{i} N_{i} \frac{d m_{i}^{2}}{d h} \int \frac{d^{3} p}{(2 \pi)^{3} 2 E} \delta f_{i}(\vec{p}, z)=0, \\
E_{s}(z) \equiv & -s^{\prime \prime}(z)+\frac{d V_{\mathrm{eff}}\left(h, s ; T_{+}\right)}{d s} \\
& +\sum_{i} N_{i} \frac{d m_{i}^{2}}{d s} \int \frac{d^{3} p}{(2 \pi)^{3} 2 E} \delta f_{i}(\vec{p}, z)=0,
\end{aligned}
$$

where $z$ is the direction normal to the wall that is to a good approximation planar by the time it has reached its terminal velocity. We use a sign convention, where the wall is moving to the left, so that $z>0$ corresponds to the broken phase. The sum is over all the relevant species coupled to $h$ or $s$ in the plasma with $N_{i}$ and $m_{i}$, respectively, denoting the number of degrees of freedom and the field-dependent mass of the corresponding species, and $\delta f_{i}$ the deviation from equilibrium of its distribution function. All the temperature-dependent quantities appearing in these equations are evaluated at $T_{+}$, which is the plasma's temperature just in front of the wall. We calculate $T_{+}$in Appendix B using the method described in Ref. [98], and $\delta f_{i}$ will be computed in Sec. IVA.

The terms in Eqs. (22) with $\delta f_{i}$ represent the friction ${ }^{4}$ of the plasma on the wall that leads to a terminal wall speed $v_{w}<1$, unless the friction is too small and the wall runs away to speeds close to that of light. Following previous work, we take the dominant sources of friction to be from the top quark $(i=t)$ and electroweak gauge bosons $(i=W)$, neglecting the contributions to friction from the Higgs itself and from the singlet. This approximation is bolstered by the smaller number of degrees of freedom $N_{h}=N_{s}=1$ compared to $N_{t}=12$ and $N_{W}=9$, as well as the smallness of the Higgs self-coupling $\lambda_{h}$ and the nottoo-large values of the cross-coupling $\lambda_{h s}$ that will be favored in the subsequent analysis. Then the friction term for the $s$ equation of motion vanishes since $s$ couples only to itself and to the Higgs, apart from its suppressed dimension-five coupling to $t$. This allows for some simplification in the following procedure.

In Ref. [64], a similar study of the present model was done, where no a priori restriction of the wall shape was assumed, but it was found that the actual shapes conform to a very good approximation to the tanh profiles

$$
\begin{aligned}
& h(z)=\frac{h_{0}}{2}\left[1+\tanh \left(z / L_{h}\right)\right], \\
& s(z)=\frac{s_{0}}{2}\left[1-\tanh \left(z / L_{s}+\delta\right)\right],
\end{aligned}
$$

where $h_{0}$ and $s_{0}$ are, respectively, the VEVs of the $h$ and $s$ fields in the broken and unbroken phases. Hence we adopt the ansatz (23), which allows the singlet and Higgs wall profiles to have different widths, and to be offset from each other by a distance $L_{s} \delta$. The $s$ field's VEV is taken to be the usual one evaluated at $T_{+}$, which solves the equation $d V_{\text {eff }}\left(0, s ; T_{+}\right) /\left.d s\right|_{s=s_{0}}=0$. The situation is more complicated for the $h$ field, for which the Higgs VEV should be evaluated at $T_{-}$, the plasma's temperature behind the wall. Since we are fixing a constant temperature $T_{+}$in the potential, the change in the effective action due to the shift in the background temperature must be accounted for by the perturbation in the broken phase. As a consequence we are choosing $h_{0}$ so that it solves the equation

$$
\begin{aligned}
& \left.\left(\frac{d V_{\mathrm{eff}}\left(h, 0 ; T_{+}\right)}{d h}+\sum_{i} N_{i} \frac{d m_{i}^{2}}{d h} \int \frac{d^{3} p}{(2 \pi)^{3} 2 E} \delta f_{i}(\vec{p}, z)\right)\right|_{h=h_{0}, z \rightarrow \infty} \\
& =0 .
\end{aligned}
$$

\footnotetext{
${ }^{4}$ The term "friction" is strictly speaking not correct, but we adopt this commonly used terminology. More accurately, the last terms in (22) represent the additional pressure created by the outof-equilibrium perturbations, which modify the effective action in the same way as the usual thermal excitations.
} 
This choice guarantees that the Higgs EOM is satisfied far behind the wall. We will estimate the uncertainty of our results due to this approximation in Sec. VID.

To approximately solve the Higgs EOM, one can define two independent moments $M_{1,2}$ of $E_{h}(z)$, and assume that they both vanish at the optimal values of $v_{w}$ and $L_{h}$. A convenient choice is [58]

$$
\begin{gathered}
M_{1} \equiv \int d z E_{h}(z) h^{\prime}(z)=0, \\
M_{2} \equiv \int d z E_{h}(z)\left[2 h(z)-h_{0}\right] h^{\prime}(z)=0 .
\end{gathered}
$$

These also have intuitive physical interpretations that naturally distinguish them as good predictors of the wall speed and thickness, respectively. $M_{1}$ is a measure of the net pressure on the wall so that Eq. (25) can be interpreted as the requirement that a stationary wall should have a vanishing total pressure; nonvanishing $M_{1}$ would cause it to accelerate. Therefore, one expects that Eq. (25) principally determines the wall speed $v_{w}$, while depending only weakly on the thickness $L_{h}$. With our sign convention, $M_{1}$ can be interpreted as the pressure in front of the wall minus the pressure behind it, so that $M_{1}>0$ corresponds to a net force slowing down the wall. On the other hand, $M_{2}$ is a measure of the pressure gradient in the wall. If nonvanishing, it would lead to compression or stretching of the wall, causing $L_{h}$ to change. Hence Eq. (26) mainly determines $L_{h}$ and depends only weakly on $v_{w}$. The two equations are approximately decoupled, facilitating their numerical solution. This is illustrated in Fig. 4, which shows the dependence of $M_{1}$ and $M_{2}$ on $v_{w}$ and $L_{h}$.

We chose a different approach to determine the singlet wall parameters $L_{s}$ and $\delta$. Instead of solving moment equations analogous to (25) and (26), one can determine their values by minimizing the $s$ field action

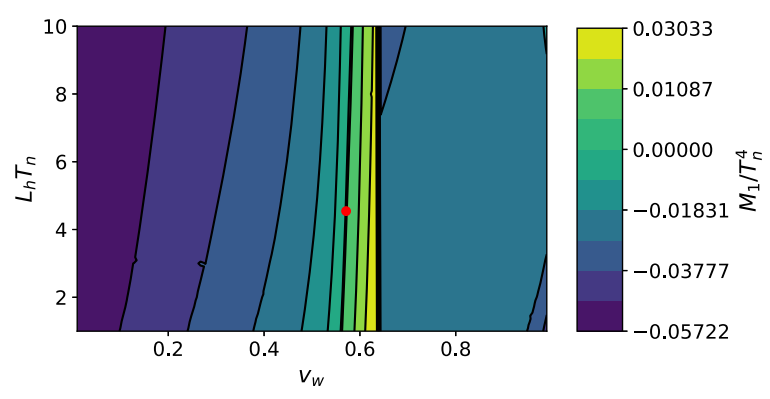

(a)

$$
\begin{aligned}
S\left(L_{s}, \delta\right) & =\int d z\left\{\frac{1}{2}\left(s^{\prime}\right)^{2}+\left[V_{\mathrm{eff}}\left(h, s, T_{+}\right)-V_{\mathrm{eff}}\left(h, s^{*}, T_{+}\right)\right]\right\} \\
& =\frac{s_{0}^{2}}{6 L_{s}}+\int d z\left[V_{\mathrm{eff}}\left(h, s, T_{+}\right)-V_{\mathrm{eff}}\left(h, s^{*}, T_{+}\right)\right],
\end{aligned}
$$

with respect to $L_{s}$ and $\delta$. Here $s^{*}$ is a field configuration with arbitrary fixed parameters $L_{s}^{*}$ and $\delta^{*}$ that we choose to be $L_{s}^{*}=L_{h}$ and $\delta^{*}=0$. The second term is just a constant, but it allows for the convergence of the integral by canceling the contributions of $V_{\text {eff }}$ at $z \rightarrow \pm \infty$. This method has the advantage that it does not depend on any arbitrary choice of moments, and it is more efficient to numerically minimize the function of two variables than to solve the system of equations for the moments of the EOMs.

\section{A. Transport equations for fluid perturbations}

The final step toward the complete determination of the velocity and the shape of the wall is to compute the distribution functions' deviations from equilibrium $\delta f_{i}$ by solving the Boltzmann equation for each relevant species in the plasma. The method of approximating the full Boltzmann equation by a truncated set of coupled fluid equations was originally carried out in Ref. [52] for the regime of slowly-moving walls (see also Ref. [58]). This approach was recently improved in Ref. [66] in order to be able to treat wall speeds close to or exceeding the speed of sound, consistently. We briefly summarize the formalism, which we use in the present study.

The out-of-equilibrium distribution function can be parametrized in the wall frame as

$$
f=\frac{1}{\exp \left[\beta \gamma\left(E-v_{+} p_{z}\right)(1-\delta \tau)-\mu\right] \pm 1}+\delta f_{u},
$$

where $\beta=1 / T_{+}$and the \pm is + for fermions and - for bosons. Note that $\delta \tau$ and $\mu$ are the dimensionless

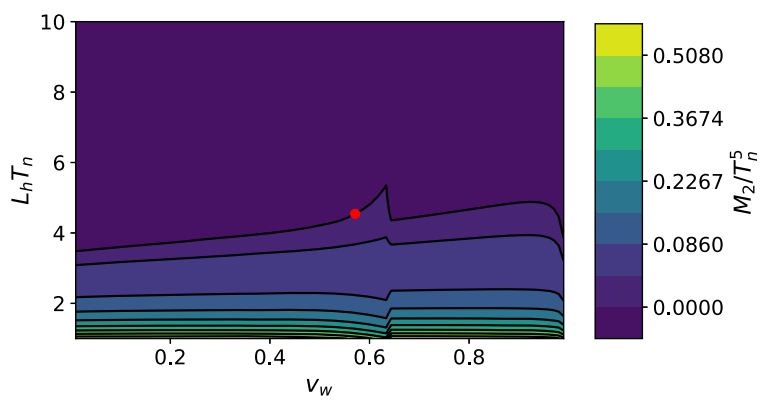

(b)

FIG. 4. Moments of the Higgs EOM (a) $M_{1}$ and (b) $M_{2}$ as a function of the wall velocity $v_{w}$ and the Higgs wall width $L_{h}$ for a model with parameters $\lambda_{h s}=1, \lambda_{s}=1$, and $m_{s}=130 \mathrm{GeV}$. The red dot is the solution of Eqs. (25) and (26). As expected, $M_{1}$ is roughly independent of $L_{h}$, while $M_{2}$ depends mainly on $L_{h}$. The moments are discontinuous at $v_{w} \approx 0.63$ because this corresponds (for this specific model) to the boundary between hybrid and detonation walls, where $v_{+}$and $T_{+}$are discontinuous. 
temperature and chemical potential perturbations from equilibrium, and $\delta f_{u}$ is a velocity perturbation, whose form is unspecified, but is constrained by $\int d^{3} p \delta f_{u}=0$. By assuming that the perturbations are small, one can expand $f$ to linear order in $\mu, \delta \tau$ and the velocity perturbation $\delta f_{u}$ to obtain

$$
\delta f \approx \delta f_{u}-f^{\prime}\left[\mu+\beta \gamma \delta \tau\left(E-v_{+} p_{z}\right)\right],
$$

with

$$
f^{\prime}=\left.\frac{d}{d X} \frac{1}{e^{X} \pm 1}\right|_{X=\beta \gamma\left(E-v_{+} p_{z}\right)} .
$$

In practice, all the perturbations and the variation of temperature across the wall are generally below $10 \%$, so the linearization of the Boltzmann equation is justified.

To simplify the problem, one models the plasma as being made of three different species: the top quark, the $W$ bosons (shorthand for $W^{ \pm}$and $Z$ ), and a background fluid, which includes all the remaining degrees of freedom. It is convenient to write the velocity perturbation as $u \propto$ $\int d^{3} p\left(p_{z} / E\right) \delta f_{u}$ when constructing the moments of the linearized Boltzmann equation. By taking three such moments, using the weighting factors $1, E$, and $p_{z} / E$, the perturbations are determined by transport equations

$$
\begin{gathered}
A q^{\prime}+\Gamma q=S, \\
q_{\mathrm{bg}}^{\prime}=-\tilde{A}_{\mathrm{bg}}^{-1}\left(\Gamma_{\mathrm{bg}, t} q_{t}+\Gamma_{\mathrm{bg}, W} q_{W}\right),
\end{gathered}
$$

where prime denotes $d / d z, q_{i}=\left(\mu_{i}, \delta \tau_{i}, u_{i}\right)^{\top}, q=\left(q_{W}^{\top}, q_{t}^{\top}\right)^{\top}$, the $\Gamma$ matrices are collision terms, and $S$ is the source term, whose definitions, as well as those of the matrices $A, \Gamma, \tilde{A}_{\mathrm{bg}}^{-1}$, $\Gamma_{\mathrm{bg}, t}, \Gamma_{\mathrm{bg}, W}$, can be found in Ref. [66]. If $A$ and $\Gamma$ were independent of $z$, one could use the Green's function method to solve Eq. (31); however, $A$ is a function of $m_{i}(z) / T$. To deal with this dependence on $z$, we discretize space, $z \rightarrow$ $z_{0}+n \Delta z$ with $n=0, \ldots, N-1$, and Fourier transform Eq. (31),

$$
\begin{aligned}
& \frac{2 \pi i}{\Delta z}\left(\frac{k}{N}-\left\lfloor\frac{2 k}{N}\right\rfloor\right) \tilde{q}_{k}+\frac{1}{N} \sum_{l=0}^{N-1} \widetilde{\left(A^{-1} \Gamma\right)_{(k-l) \bmod N} \tilde{q}_{l}} \\
& \quad=\left(\widetilde{A^{-1} S}\right)_{k}, \quad k=0, \ldots, N-1,
\end{aligned}
$$

where the tilde denotes the discrete Fourier transform. This is a linear system that is straightforward to numerically solve for $\tilde{q}_{k}$. Once $\tilde{q}_{k}$ is known, it can be transformed back and interpolated to obtain $q(z)$. Equation (32) can then be integrated using a Runge-Kutta algorithm.

Finally, one can substitute Eq. (29) into the Higgs EOM (22) to express the friction in terms of the fluid perturbations $\mu_{i}, \delta \tau_{i}$, and $u_{i}$. This leads to the result

$$
\begin{aligned}
& \int \frac{d^{3} p}{(2 \pi)^{3} 2 E} \delta f_{i} \\
& \quad=\frac{T_{+}^{2}}{2}\left[C_{0}^{1,0} \mu_{i}+C_{0}^{0,0}\left(\delta \tau_{i}+\delta \tau_{\mathrm{bg}}\right)+D_{v}^{0,-1}\left(u_{i}+u_{\mathrm{bg}}\right)\right]
\end{aligned}
$$

where the functions $C_{v}^{m, n}$ and $D_{v}^{m, n}$ can be found in Ref. [66].

\section{COSMOLOGICAL SIGNATURES}

We have now established the machinery needed to compute all the relevant properties of the first-order phase transition bubbles, starting from the fundamental parameters of the microscopic Lagrangian. In this section we describe how to apply these results for the estimation of GW spectra and the baryon asymmetry.

\section{A. Gravitational Waves}

We follow the methodology of Refs. $[7,67,68,98,100]$ to estimate future gravitational wave detectors' sensitivity to the GW signals that can be produced by a first-order electroweak phase transition in the models under consideration. The GW spectrum $\Omega_{\mathrm{gw}}(f)$ is the contribution per frequency octave to the energy density in gravitational waves, i.e., $\int \Omega_{\mathrm{gw}} d \ln f$ is the fraction of energy density compared to the critical density of the Universe. The spectrum gets separate contributions from the scalar fields, sound waves in the plasma, and magnetohydrodynamical turbulence created by the phase transition:

$$
\Omega_{\mathrm{gw}}(f)=\Omega_{\phi}(f)+\Omega_{\mathrm{sw}}(f)+\Omega_{m}(f) .
$$

Each of these contributions depends on the wall velocity $v_{w}$, the supercooling parameter $\alpha$ [Eq. (21)], and the inverse duration of the phase transition, defined as

$$
\beta=\left.H\left(T_{n}\right) T_{n} \frac{d}{d T} \frac{S_{3}}{T}\right|_{T=T_{n}} .
$$

Another useful quantity is the mean bubble separation, which can be written in terms of $v_{w}$ and $\beta$ as [7]

$$
R=\frac{(8 \pi)^{1 / 3}}{\beta} \max \left[c_{s}, v_{w}\right] .
$$

It has been shown in Ref. [59] that interactions with gauge bosons prevent the wall from running away indefinitely towards $\gamma \rightarrow \infty$. In that case, the contribution from the scalar fields has been shown to be negligible. Furthermore, the estimates for the magnetohydrodynamical turbulence are very uncertain and sensitive to the details of the phase transition dynamics [101] and are expected to be much smaller than the contribution from sound waves. Hence, we 
consider only the effects from the latter and set $\Omega_{m}(f)=\Omega_{\phi}(f)=0$. For convenience, we reproduce the numerical fits of the GW spectra derived in Refs. $[7,67,68,98,100]$ in Appendix C.

We will use these predictions with respect to four proposed space-based GW detectors: LISA [102], AEDGE [11], BBO [103] and DECIGO [9]. A successful GW detection depends upon having a large enough signalto-noise ratio [104],

$$
\mathrm{SNR}=\sqrt{\mathcal{T} \int_{f_{\min }}^{f_{\max }} d f\left[\frac{\Omega_{\mathrm{gw}}(f)}{\Omega_{\mathrm{sens}}(f)}\right]^{2}},
$$

where $\Omega_{\text {sens }}(f)$ denotes the sensitivity of the detector, ${ }^{5}$ and $\mathcal{T}$ is the duration of the mission. The sensitivity curves for the detector LISA, BBO, and DECIGO were obtained from Ref. [105]. Whenever SNR is greater than a given threshold $\mathrm{SNR}_{\text {thr }}$, we conclude that the signal can be detected. In general, this threshold can depend upon the configuration of the detector. For all the experiments, we take $\mathrm{SNR}_{\text {thr }}=10$ and $\mathcal{T}=1.26 \times 10^{8} \mathrm{~s}$. In the following, $\mathrm{SNR}_{\max }$ will designate the maximum signal-to-noise ratio detected by one of the detectors:

$\mathrm{SNR}_{\max } \equiv \max \left[\mathrm{SNR}_{\mathrm{LISA}}, \mathrm{SNR}_{\mathrm{AEDGE}}, \mathrm{SNR}_{\mathrm{BBO}}, \mathrm{SNR}_{\mathrm{DECIGO}}\right]$.

While $\Omega_{\text {sens }}(f)$ can be obtained from the noise spectrum of a detector, it is not practical to compare it to the GW spectrum directly; one needs to compute the SNR to determine if a signal is detectable. A useful tool for visualizing the sensitivity of a detector is the peak-integrated sensitivity curve (PISC) defined in Refs. [106-108], which is a generalization of the power-law sensitivity curve [109]. The main advantage of the former is that it does not assume a power-law spectrum, hence it conserves all the information about the SNR. In the simple case where one considers the contribution from only one GW source, the PISC can be obtained by factorizing the GW spectrum as

$$
\Omega_{\mathrm{gw}}(f)=\Omega_{p} S\left(f, f_{p}\right),
$$

where $f_{p}$ and $\Omega_{p}=\max \left[\Omega_{\mathrm{gw}}(f)\right]$ are the peak frequency and GW amplitude, and $S$ is a function that parametrizes the spectrum's shape, with a maximum at $f=f_{p}$ and $S\left(f_{p}, f_{p}\right)=1$. One can then write the SNR as

$$
\mathrm{SNR}=\mathrm{SNR}_{\mathrm{thr}} \frac{\Omega_{p}}{\Omega_{\mathrm{PISC}}\left(f_{p}\right)},
$$

\footnotetext{
${ }^{5}$ For AEDGE, we use the envelope of minimal strain that can be achieved by each resonance, with its width scaled to approximate $\Omega_{\text {sens }}(f)$. This curve is expected to reproduce the correct SNR up to about $10 \%$.
}

with the PISC

$\Omega_{\mathrm{PISC}}\left(f_{p}\right)=\mathrm{SNR}_{\mathrm{thr}}\left[\mathcal{T} \int_{f_{\min }}^{f_{\max }} d f\left(\frac{S\left(f, f_{p}\right)}{\Omega_{\mathrm{sens}}(f)}\right)^{2}\right]^{-1 / 2}$

By construction, any GW signal that peaks above the PISC has $\mathrm{SNR}>\mathrm{SNR}_{\mathrm{thr}}$ and can, therefore, be detected.

\section{B. Baryogenesis}

The mechanism of electroweak baryogenesis is sensitive to the speed and shape of the bubble wall during the phase transition. In most previous studies, these quantities were treated as free parameters to be varied, but in this work we have already derived them, as was discussed in Sec. IV. An important requirement for EWBG is to avoid the washout, by baryon-violating sphaleron interactions, of the generated asymmetry inside the bubbles of the broken phase, once they have formed. This leads to the well-known constraint [110]

$$
\frac{v_{n}}{T_{n}}>1.1
$$

which was derived within the SM for low Higgs masses where a first order EWPT was possible. The bound can be slightly higher (up to 1.2) in singlet-extended models [111], depending upon the parameters, due to the sphaleron energy being modified. Here we adopt the SM constraint (43); we checked that taking the more stringent bound 1.2 removes $\sim 5 \%$ of viable models in the scan over parameter space to be described below.

Near the bubble wall, $C P$-violating processes associated with the effective interaction in Eq. (2) give rise to perturbations of the plasma that result in a local chemical potential $\mu_{B_{L}}$ for left-handed baryons, which by imposing the chemical equilibrium of strong-sphaleron interactions, is related to those of the $t_{L}, t_{R}^{c}$, and $b_{L}$ quarks by

$$
\mu_{B_{L}}=\frac{1}{2}\left(1+4 K_{1}^{t}\right) \mu_{t}+\frac{1}{2}\left(1+4 K_{1}^{b}\right) \mu_{b}-2 K_{1}^{t} \mu_{t^{c}},
$$

where the $K_{1}^{a}$ functions were defined in [112] $\left(K_{1}^{a}=D_{0}^{a}\right.$ in the notation of [65]). The $\mu_{B_{L}}$ potential biases sphalerons, leading to baryon number violation, whose associated Boltzmann equation can be integrated to obtain the baryon to photon ratio, ${ }^{6}$

$$
\eta_{b}=\frac{405 \Gamma_{\mathrm{sph}}}{4 \pi^{2} v_{w} \gamma_{w} g_{*} T} \int d z \mu_{B_{L}} f_{\mathrm{sph}} e^{-45 \Gamma_{\mathrm{sph}}|z| / 4 v_{w}},
$$

where $f_{\text {sph }}$ quantifies the diminution of the sphaleron rate in the broken phase $[113,114]$. The most challenging step for

\footnotetext{
${ }^{6}$ The extra factor of $\gamma_{w}=1 / \sqrt{1-v_{w}^{2}}$ in the denominator was pointed out by Ref. [65].
} 
the computation of EWBG is in the determination of the chemical potentials $\mu_{t_{L}}, \mu_{t_{R}^{c}}$, and $\mu_{b_{L}}$ appearing in Eq. (44). They satisfy fluid equations resembling the network (31) and (32), except that the potentials relevant for EWBG are $C P$ odd, whereas those determining the wall profiles are $C P$ even.

The $C P$-odd transport equations have been discussed extensively in the literature, leading to two schools of thought as to how best to compute the source term for the $C P$ asymmetries. These are commonly known as the VEVinsertion [115,116] or WKB (semiclassical) [117-122] methods, respectively. A detailed discussion and comparison of the two approaches was recently given in Ref. [65], which quantified the well-known fact that the VEVinsertion source tends to predict a larger baryon asymmetry than the WKB source by a factor of $\sim 10$. In the present work we adopt the WKB approach, which was updated in Ref. [65] to allow for consistently treating walls moving near or above the sound speed. In addition, that reference computed the source term arising from the same effective interaction (2) as in the present model, so we can directly adopt the $C P$-odd fluid equations studied there.

\section{MONTE CARLO RESULTS}

To study the properties of the phase transition, we performed a scan over the parameter space of the models, imposing several constraints. We found that variations in $\lambda_{s}$ do not qualitatively change the results, prompting us to initially fix its value at $\lambda_{s}=1$, leaving $\lambda_{h s}$ and $m_{s}$ as the free scalar potential parameters. We will first discuss this slice of parameter space and later consider the quantitative dependence on $\lambda_{s}$. We also chose $\Lambda=540 \mathrm{GeV}$, which is conservative since there are no collider constraints on its value for singlet masses in the region $m_{s}=[110,160] \mathrm{GeV}$. Recall that $\Lambda$ is important for the determination of the baryon asymmetry $\eta_{b}$, which is expected to scale roughly as $1 / \Lambda$. Finally, in order to prevent Higgs invisible decays, we imposed $m_{s}>m_{h} / 2$.

We used a Markov Chain Monte Carlo algorithm to efficiently explore the regions of parameter space having desired phase transition properties. Starting with an initial model satisfying the sphaleron bound (43), one generates a new trial model by randomly varying the parameters $\lambda_{i}$ by small increments $\delta_{i}$. The trial model is added to the chain using a conditional probability

$$
P=\min \left[\frac{v_{n} / T_{n}}{1.1}, 1\right]
$$

that favors models having strong first-order phase transitions, and for which a solution to the nucleation condition (20) can be found. We adjust the $\delta_{i}$ so that roughly half of the models are kept in successive trials, with larger values of $\delta_{i}$ being more likely to result in a rejection.
This procedure yielded 842 models with strong phase transitions, of which 712 were amenable to finding solutions for the moment equations (25) and (26). Our analysis typically works for $\gamma \lesssim 10$; for faster walls, the algorithm for determining the wall properties becomes numerically unstable and does not yield reliable results. This is due to the large $(500 \times 500)$ matrix $\left(\widetilde{A^{-1} \Gamma}\right)$ of Eq. (33), becoming singular as $v_{w} \rightarrow 1$. It is, therefore, difficult to determine the type of solution of the 130 remaining models using our methodology alone: they could either stabilize at ultrarelativistic speeds, or (from a naive perspective-see below) run away indefinitely towards $\gamma \rightarrow \infty$. The value of the baryon asymmetry should not be affected by this ambiguity since it is negligible for $v_{w} \approx 1$. The $\mathrm{GW}$ spectrum produced during the phase transition is sensitive to this distinction since runaway walls have a non-negligible fraction of their energy stored in the wall, while for nonrunaway walls, the energy gets dissipated into the plasma, so the fraction of energy in the wall becomes negligible. This ambiguity can be lifted using the result of Ref. [59], which found that in the limit $\gamma \rightarrow \infty$, interactions between gauge bosons and the wall create a pressure proportional to $\gamma$, preventing it from running away. ${ }^{7} \mathrm{We}$, therefore, assume that the 130 models without a solution to the moment equations (25) and (26) correspond to nonrunaway walls with $v_{w} \approx 1$. The results of this scan, showing the calculated wall velocity, signal-to-noise ratio of gravity waves observable by at least one of the proposed experiments (LISA, AEDGE, BBO, or DECIGO), and the predicted baryon asymmetry (in units of the observed value) are presented in Fig. 5, in the plane of $\lambda_{h s}$ versus $m_{s}$.

\section{A. Deflagration versus detonation solutions}

A striking feature of these results is that all the detonation solutions have $v_{w} \approx 1 .^{8}$ We have tested that this is not specific to the choice of fixed parameter values, but also holds for all models having $0.01<\lambda_{s}<8$ and $\Lambda>110 \mathrm{GeV}$; hence it seems to be a general property of phase transitions in the $Z_{2}$-symmetric singlet framework. One can understand this behavior by considering the net pressure opposing the wall's expansion $M_{1}$ [recall Eq. (25) and (26)], as a function of the wall velocity, as illustrated in Fig. 6. It shows how $M_{1}$ differs when evaluated with the appropriate quantities $v_{+}, T_{+}$rather than the incorrect ones $v_{w}, T_{n}$. Using the latter, we would find no solution to the equation $M_{1}=0$ for the exemplary model used in Fig. 6

\footnotetext{
${ }^{7}$ More recently, the authors of Ref. [60] have carried out an allorders resummation at leading-log accuracy, finding that the pressure is, in fact, proportional to $\gamma^{2}$ for fast-moving walls.

${ }^{8}$ Strictly speaking there are models with $v_{w}<1$ detonation solutions but these always have another solution at a lower velocity corresponding to a deflagration or hybrid wall. Then only the latter solution is physically relevant since the bubble is created at $v_{w}=0$ and accelerates until it reaches the solution with the lowest velocity.
} 


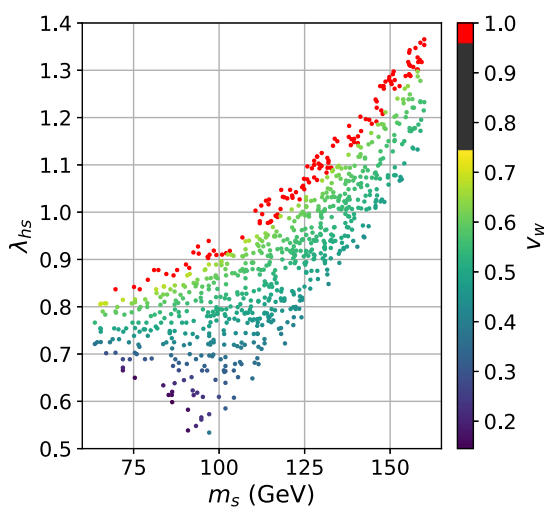

(a)

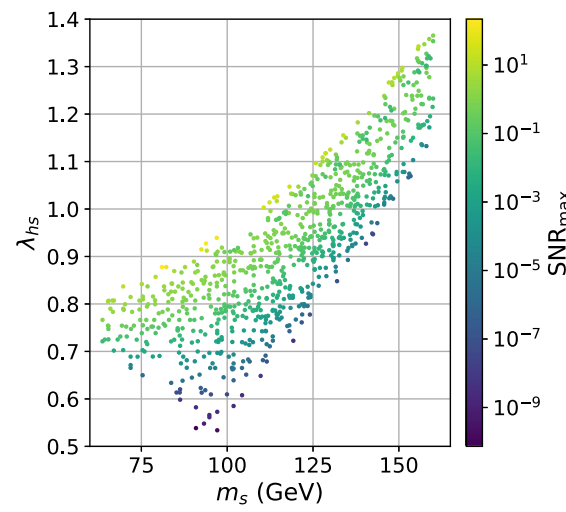

(b)

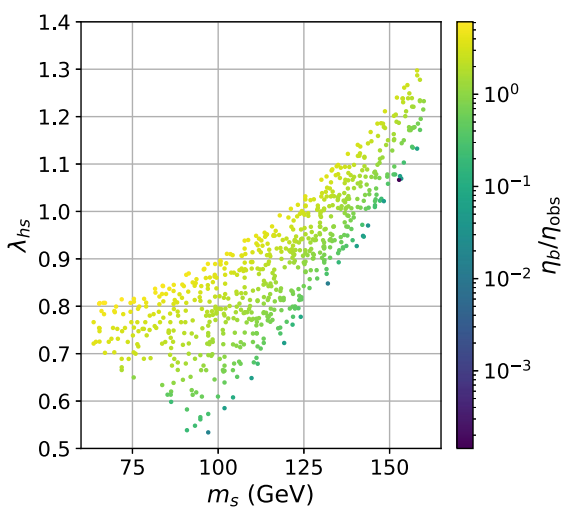

(c)

FIG. 5. Scan of the parameter space with $\lambda_{s}=1$ and $\Lambda=540 \mathrm{GeV}$. The colors represent (a) the terminal wall velocity $v_{w}$, (b) the maximum signal-to-noise ratio of gravitational waves that could be detected by either LISA, AEDGE, BBO, or DECIGO and (c) the baryon asymmetry (in units of the observed value) produced by the phase transition. The red dots in (a) correspond to detonation solutions with $v_{w} \approx 1$, and the latter are not included in (c) since they are expected to produce a negligible baryon asymmetry (see text).

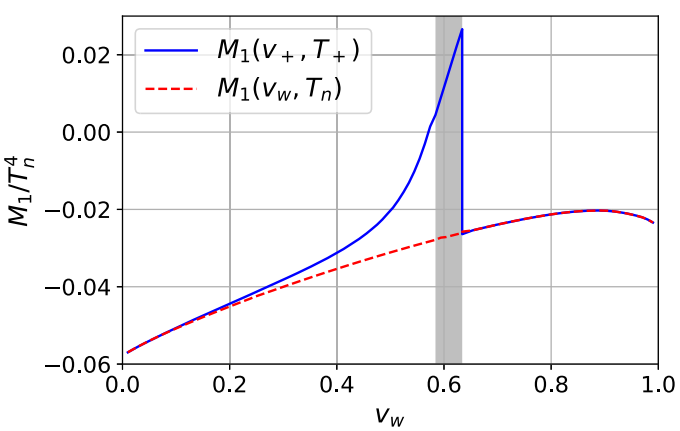

(a)

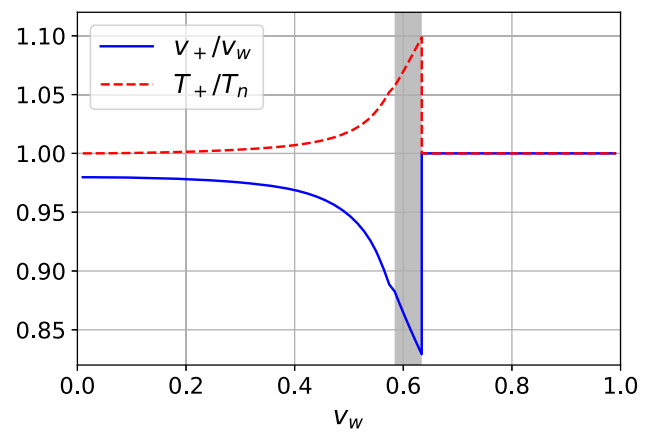

(b)

FIG. 6. Left (a): Pressure on the wall $M_{1}$ as a function of the wall velocity $v_{w}$. The solid (dashed) line corresponds to the pressure evaluated at the velocity $v_{+}\left(v_{w}\right)$ and the temperature $T_{+}\left(T_{n}\right)$. Right (b): Relation between the naive variables $v_{w}, T_{n}$, and the ones relevant for evaluating $M_{1}$, namely $v_{+}$and $T_{+}$. Both plots were obtained using the parameters $m_{s}=130 \mathrm{GeV}, \lambda_{h s}=\lambda_{s}=1$, and $L_{h}=5 / T_{n}$. The shaded region corresponds to hybrid wall solutions characterized by $c_{s}<v_{w}<\xi_{J}$.

and would then incorrectly conclude that it satisfies $v_{w} \approx 1$. The relevant quantities are those measured right in front of the wall, $v_{+}$and $T_{+}$. The speed $v_{+}$is smaller than $v_{w}$ for $v_{w}<\xi_{J}$, which would lower the pressure against the wall $\left(\xi_{J}\right.$ is the Jouguet velocity, defined as the smallest velocity a detonation solution can have). However, in the same region, the temperature $T_{+}$is larger than $T_{n}$, which causes the pressure to increase. The latter effect turns out to dominate over the former. Indeed, the actual pressure, represented by the solid blue line in Fig. 6, increases much more rapidly than $M_{1}\left(v_{w}, T_{n}\right)$ close to the speed of sound. This qualitative difference allows for a solution to $M_{1}=0$, which would have been missed if we had used the naive quantities $v_{w}$ and $T_{n}$.

We find that the previous statements apply quite generally: for all models, $T_{+}>T_{n}$ when $v_{w}<\xi_{J}$, and this always leads to a much higher pressure on the wall, even if the difference between $T_{+}$and $T_{n}$ is quite small; the pressure barrier at $v_{w}=\xi_{J}$ is always greater than the maximum possible value for a detonation solution. Therefore, if the phase transition is strong enough to overcome the pressure barrier at $\xi_{J}$, the solution becomes a detonation, but the pressure in the region $v_{w}>\xi_{J}$ is never enough to prevent it from accelerating towards $v_{w} \approx 1$. If the phase transition is weaker, the pressure barrier is high enough to impede the detonation, and it becomes a deflagration or hybrid solution.

The wall thickness and speed for the models with deflagration ${ }^{9}$ solutions are shown in Fig. 7, which demonstrates that the behaviors for subsonic (deflagration) and supersonic (hybrid) walls are qualitatively different. Subsonic walls generally have $v_{+} \approx v_{w}$, which is expected since the fluid should not be strongly perturbed by a slowly

\footnotetext{
${ }^{9}$ Henceforth, we take "deflagration" to also include hybrid solutions.
} 


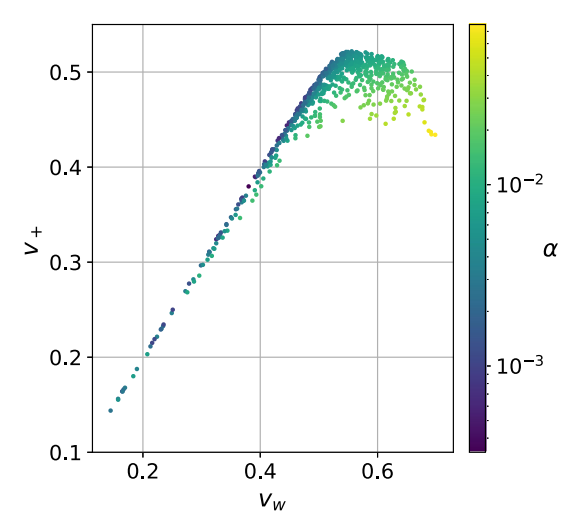

(a)

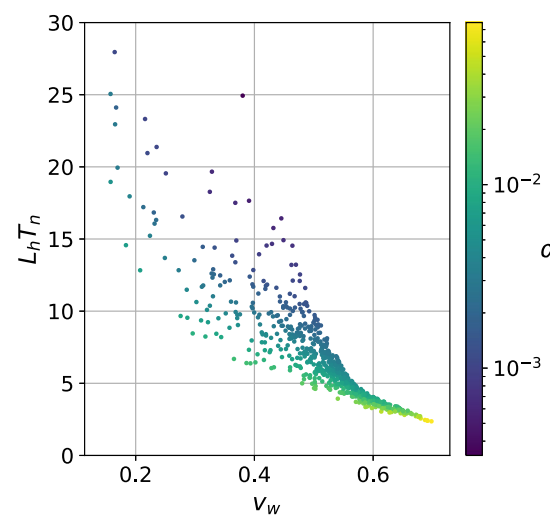

(b)

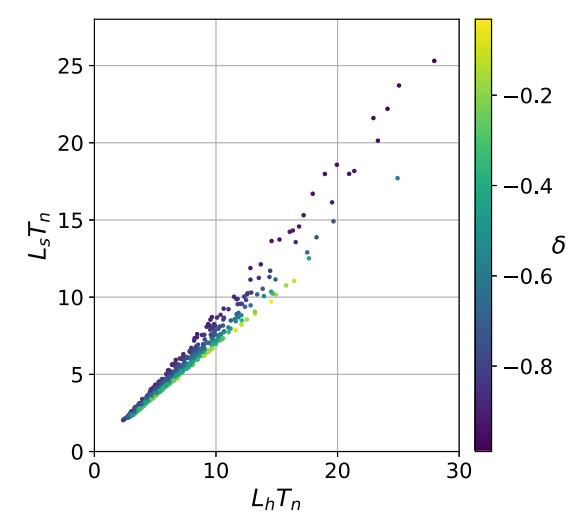

(c)

FIG. 7. Shape and velocity of the deflagration solutions. (a) Correlation between the wall velocity $v_{w}$ and the fluid velocity in front of the wall $v_{+}$; (b) dimensionless wall width $L_{h} \times T_{n}$ versus $v_{w}$; and (c) correlation of the $s$ and $h$ wall widths. Colors indicate the supercooling parameter $\alpha$ [Eq. (21)] in (a) and (b), or the wall offset $\delta$ [Eq. (23)] in (c).

moving wall. The wall width is not uniquely determined by $v_{w}$, but there exists a clear correlation, with slower walls being thicker. For supersonic cases, the correlation between $v_{+}$and $v_{w}$ gets inverted: higher wall velocity leads to lower $v_{+}$. The wall width becomes uniquely determined by $v_{w}$, and the relation between these two variables is to a good approximation linear. One observes that stronger phase transitions, quantified by higher values of $\alpha$, generally produce faster and thinner walls. Even for the strongest transitions, our solutions still have wall thickness $L T \gtrsim 3$. Since the semiclassical force mostly affects particles with momenta $\left\langle k_{z}\right\rangle \sim T$, we find $L\left\langle k_{z}\right\rangle \gtrsim 3$, so that the semiclassical approximation is still valid. In fact, the semiclassical picture has been shown to remain valid for surprisingly narrow walls [123], working very well for $L\left\langle k_{z}\right\rangle \approx 4$ and still reasonably for $L\left\langle k_{z}\right\rangle \approx 2$. There is a linear correlation between the $h$ and $s$ wall widths, but the slope is not 1 ; in all cases, we find that $L_{h}>L_{s}$. The distribution of wall offset values $\delta$ is also indicated in Fig. 7(c).

\section{B. Baryogenesis and gravity wave production}

Of the 842 sampled models, 517 are able to generate the baryon asymmetry at a level large enough to agree with observations, and 20 detonation walls can produce observable gravitational waves. We found no detectable deflagration solutions. More detailed results are presented in Table II. The complementarity of the experiments considered here, with respect to the present model, can be appreciated by considering the relation between the maximum GW amplitude ${ }^{10} \max \left[\Omega_{\mathrm{gw}} h^{2}\right]$ and the frequency of this peak amplitude $f_{\max }$, as shown in Fig. 8(a). The peak frequency of the strongest detonation walls are positioned exactly in LISA's region of maximal sensitivity, while the

\footnotetext{
${ }^{10} h=0.678$ is the reduced Hubble constant defined by $H_{0}=$ $100 h \mathrm{~km} \mathrm{~s}^{-1} \mathrm{Mpc}^{-1}$ [124].
}

peak frequency of the deflagration solutions are closer to the peak sensitivity of AEDGE, DECIGO, and BBO. The complete spectrum's shape are also shown in Fig. 8(b) and 8(c) for deflagration and detonation solutions, respectively. We conclude that detonation walls could be probed by LISA, DECIGO, and BBO, but not by AEDGE.

In previous studies, where the wall velocity was considered as a free parameter, there was an expectation that baryogenesis would be less efficient with increasing $v_{w}$, whereas gravity waves would become more so. In the present study, where $v_{w}$ is not adjustable but is a derived parameter, we surprisingly find that rather than EWBG and stronger GWs being anticorrelated, instead they are positively correlated, as is illustrated in Fig. 9(a). This can be understood from the fact [see Fig. 7(b)] that $L_{h}$ is a decreasing function of $v_{w}$, which enhances EWBG. Moreover, the relevant velocity for EWBG is $v_{+}$, which is a decreasing function of $v_{w}$ for supersonic walls, and is bounded by $v_{+}<c_{s}$; this effect also enhances EWBG for fast-moving walls. The actual relation between $\eta_{b}$ and $v_{w}$ is shown in Fig. 9(b) and, at least for supersonic walls, there is a positive correlation between these two variables. Figure 9 also indicates that the supercooling parameter $\alpha$ is positively correlated with both $\eta_{b}$ and $\mathrm{SNR}_{\max }$ : stronger phase transitions generally lead to both higher GW and baryon production.

Detailed predictions for EWBG in the $Z_{2}$ symmetric model were previously made in Refs. [44,34], as opposed to merely requiring the sphaleron bound (43) to be satisfied. Comparisons with the present work are hindered by the fact that different source terms for the $C P$ asymmetry were assumed. In Ref. [44], the dimension-six coupling $i\left(y_{t} / \sqrt{2}\right)(s / \Lambda)^{2} \bar{h} t_{L} t_{R}$ was used, rather than the dimension-five coupling in Eq. (2). Moreover, a value $v_{w}=0.2$ was taken for the wall velocity, and an estimate $L_{h}=$ $v_{n} / \sqrt{8 V_{b}}$ was made for the wall width, where $v_{n}$ is the Higgs VEV at the nucleation temperature, and $V_{b}$ is the 


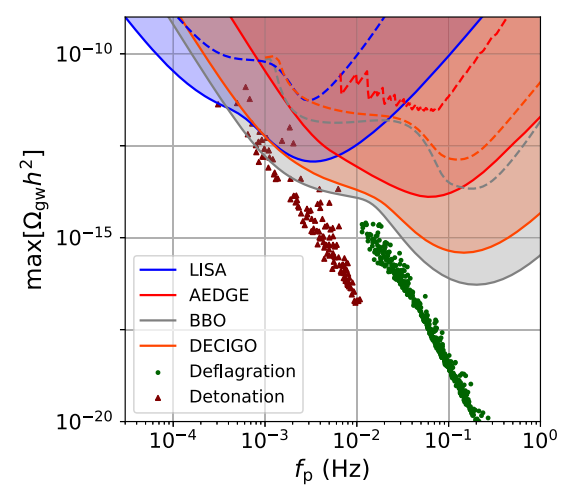

(a)

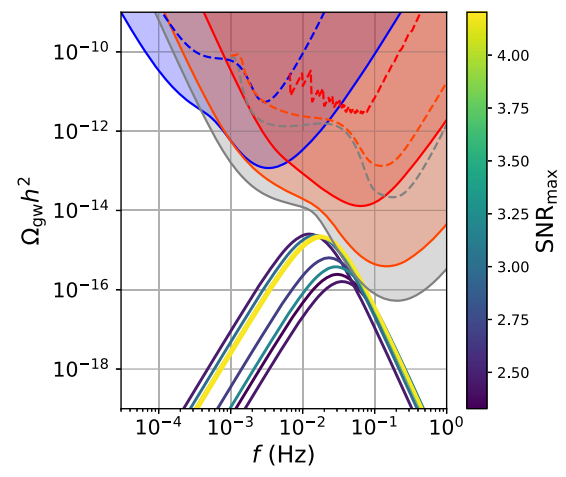

(b)

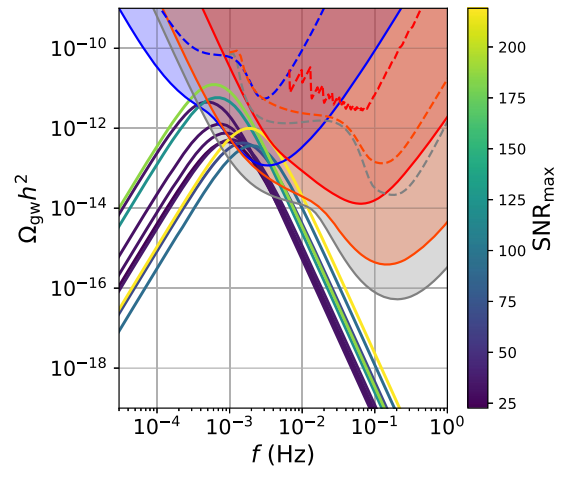

(c)

FIG. 8. (a): Maximum amplitude of GW as a function of the peak frequency $f_{p}$ with the peak-integrated sensitivity curve $\Omega_{\text {PISC }} h^{2}$ (solid line) and the sensitivity $\Omega_{\text {sens }} h^{2}$ (dashed line) of the four considered detectors. (b) and (c): Spectrum of GWs produced by the ten models with the highest $\mathrm{SNR}_{\max }$ for (b) deflagration and (c) detonation solutions.
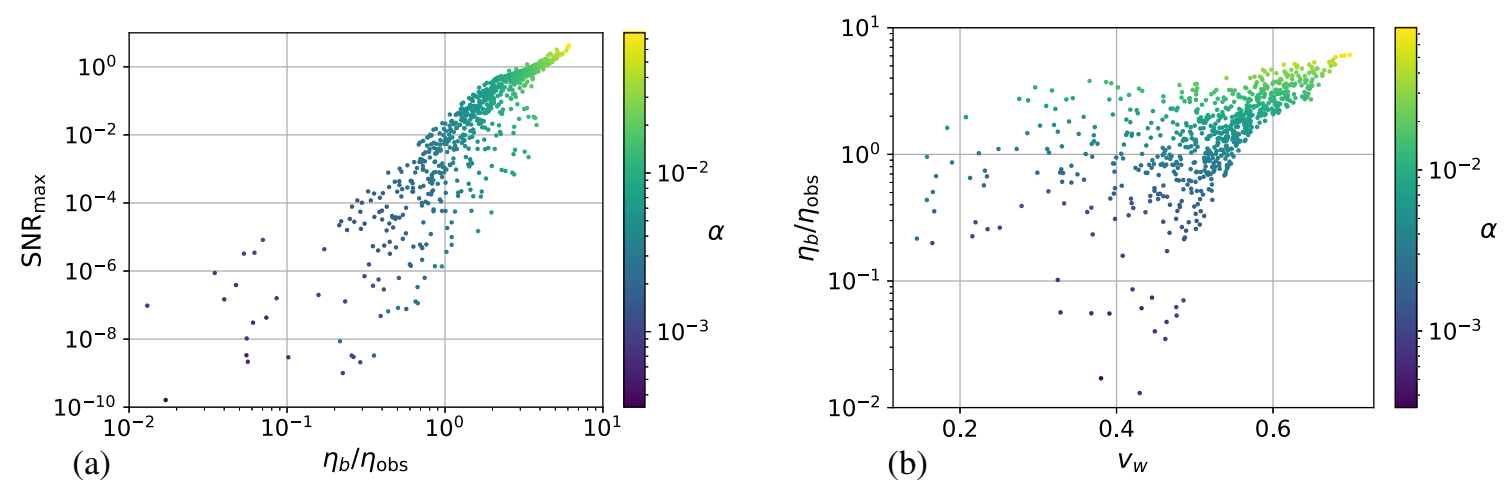

FIG. 9. (a): Relation between the $\mathrm{SNR}_{\max }$ and the baryon asymmetry produced by the phase transition. (b): Baryon asymmetry as a function of the wall velocity. Both plots only show the deflagration models.

potential barrier between the two minima. For the same potential parameters $\left(\lambda_{s}=0.1\right)$ as in [44], we find no values of $v_{w}$ below 0.43 , and our determination of $L_{h}$ is two to three times larger than the estimate in [44]. Both of these discrepancies would lead to overestimating the efficiency of EWBG, helping to explain why Ref. [44] obtains a high frequency of successful models, despite the extra suppression that should result from using a dimension-six source term.

In Ref. [34], the dimension-five coupling to leptons rather than the top quark was studied, and a different formalism (the VEV insertion approximation) for computing the $C P$ asymmetry was employed, which tends to give significantly larger estimates for the baryon asymmetry than the WKB method that we adopt [65]. For the parameters of the benchmark models taken in that paper, we find significantly higher wall velocities, $v_{w} \sim 0.6-0.7$ than the values $v_{w} \lesssim 0.1$ that were needed to match the observed baryon asymmetry there. This can be compensated by increasing the $C P$-violating phase $\phi=0.02$ assumed there by a factor of $\sim 10$. We are reanalyzing this alternative source term within the EWBG formalism used in the present paper (work in progress).

\section{Dependence on $\lambda_{s}$ and $\Lambda$}

To study the quantitative dependence on the singlet selfcoupling $\lambda_{s}$, we performed three other scans similar to the one previously described, taking $\lambda_{s}=0.01,0.1$ and 8 (the largest value being near the limit of perturbative unitarity) and $\Lambda=540 \mathrm{GeV}$. The results of these scans are summarized in Table II. We find that EWBG remains efficient for $\lambda_{s} \gtrsim 0.1$. Again, we found no deflagration walls producing detectable GWs, and no models detectable by AEDGE. These results confirm that only detonation solutions, which are not good candidates for EWBG, could be probed by $\mathrm{GW}$ detectors. Increasing $\lambda_{s}$ generally leads to stronger phase transitions, resulting in more models with successful EWBG and detectable GWs.

The value of $\Lambda$ [recall Eq. (4)] can, in principle, also have an effect on the strength of the phase transition, through the effective potential's dependence on the top quark mass. The leading thermal term added to the potential varies like 
TABLE II. Statistics from the scans performed with $\lambda_{s}=0.01,0.1,1,8$ and $\Lambda=540 \mathrm{GeV}$ and $\Lambda_{\min }$. Each entry corresponds to the percentage of models satisfying the indicated constraint. In the row for $\lambda_{s}=1$ and $\Lambda=540 \mathrm{GeV}$, the exponents (indices) correspond to the error obtained by substituting the collision matrix $\Gamma$ for $2 \Gamma(\Gamma / 2) . \Lambda_{\min }$ is the minimum value of $\Lambda$ allowed by laboratory constraints.

\begin{tabular}{|c|c|c|c|c|c|c|c|}
\hline \multirow[b]{2}{*}{$\Lambda$} & \multirow[b]{2}{*}{$\lambda_{s}$} & \multirow[b]{2}{*}{$\eta_{b} / \eta_{\mathrm{obs}}>1$} & \multicolumn{5}{|c|}{ Detonation } \\
\hline & & & Total & $\mathrm{SNR}_{\max }>10$ & $\mathrm{SNR}_{\text {LISA }}>10$ & $\mathrm{SNR}_{\mathrm{BBO}}>10$ & $\mathrm{SNR}_{\text {DECIGO }}>10$ \\
\hline \multirow{4}{*}{$540 \mathrm{GeV}$} & 0.01 & 0 & 80.5 & 2.68 & 0.8 & 2.5 & 0.27 \\
\hline & 0.1 & 10.1 & 53 & 0.89 & 0.2 & 0.89 & 0.2 \\
\hline & 1 & $61.4_{+4.6}^{-5.6}$ & $15.4_{-1.4}^{+2.4}$ & $2.38_{-0}^{+0}$ & $0.83_{-0}^{+0}$ & $2.38_{-0}^{+0}$ & $0.71_{-0}^{+0}$ \\
\hline & 8 & 73.3 & 26.4 & 6.2 & 2.81 & 6.2 & 3.16 \\
\hline \multirow{3}{*}{$\Lambda_{\min }$} & 0.1 & 21.6 & 49.3 & 1.39 & 0.69 & 1.19 & 0.4 \\
\hline & 1 & 69.6 & 18.1 & 2.21 & 0.97 & 2.07 & 0.97 \\
\hline & 8 & 85.7 & 13.8 & 3.55 & 1.01 & 3.55 & 1.52 \\
\hline
\end{tabular}

$h^{2} s^{2} T^{2} / \Lambda^{2}$, which becomes negligible at high $\Lambda$, but could significantly modify the behavior of the phase transition for $\Lambda \sim T_{n}$, resulting in a larger baryon asymmetry and GW production. We have verified that this term is already subdominant when $\Lambda=540 \mathrm{GeV}$. However, for $m_{s}>110 \mathrm{GeV}$, the weaker constraints allow for values of $\Lambda$ as low as $300 \mathrm{GeV}$, which could have an important effect on the phase transition.

To test the sensitivity to lower values of $\Lambda$, we repeated the previous scans using $\Lambda=\Lambda_{\min }\left(m_{s}\right)$, where $\Lambda_{\min }$ is given by

$$
\Lambda_{\min }\left(m_{s}\right)= \begin{cases}540 \mathrm{GeV}, & m_{s}<110 \mathrm{GeV} \\ 300 \mathrm{GeV}, & 110 \mathrm{GeV}<m_{s}<160 \mathrm{GeV}\end{cases}
$$

The results are shown in Table II. ${ }^{11}$ As one could anticipate from the relation $\eta_{b} \sim 1 / \Lambda$, EWBG is more efficient at lower values of $\Lambda$. One can also see that the number of detonation walls or walls generating detectable GWs does not change substantially, which indicates that the lower values of $\Lambda$ do not change the character of the phase transition.

\section{Theoretical uncertainties}

In Ref. [66], the integrals that determine the collision rates $\Gamma$ appearing in the Boltzmann equation network (31) and (32) were reevaluated, and it was noticed that the leading log approximation that was used in their derivation leads to theoretical uncertainties of $\mathcal{O}(1)$ in the fractional error. To study the impact of these uncertainties on our results, we recomputed the wall velocity with uniformly rescaled collision rates, $\Gamma \rightarrow 2 \Gamma$ and $\Gamma \rightarrow \Gamma / 2$. The ensuing variations of velocity $\Delta v$ and wall width $\Delta L$ are shown in Figs. 10(a) and (b), respectively. The effect on $v_{w}$ can be

\footnotetext{
${ }^{11}$ The $\lambda_{s}=0.01$ scan is omitted since all accepted models satisfy $m_{s}<110 \mathrm{GeV}$, making the results identical to those of the previous scan.
}

significant for slow walls, leading to a $\pm 40 \%$ change when $v_{w} \sim 0.2$. On the other hand, for nearly supersonic walls $v_{w} \gtrsim c_{s}$, the wall speed is quite insensitive to $\Gamma$. The variation of $L_{h}$ is generally below $5 \%$, much smaller than the corresponding variation in $\Gamma$.

This behavior is not surprising since, near the speed of sound, the pressure on the wall is mainly determined by the variation of $T_{+}$, which does not depend on $\Gamma$. Likewise, the results for the baryon asymmetry and GW production turn out to be relatively robust against variations in $\Gamma$. This is demonstrated by the error intervals in the $\lambda_{s}=1$ row of Table II. The error on the ratio of models satisfying $\eta_{b} / \eta_{\mathrm{obs}}>1$ or $\mathrm{SNR}_{i}>10$ is of order $10 \%$, which is much smaller than the range of variation in $\Gamma$.

Another source of uncertainty is the discrepancy between the temperatures computed with the Boltzmann equation (see Sec. IV A) and the conservation of the energy-momentum tensor (see Appendix B). Ideally, one should obtain $T_{+}=$ $T_{\mathrm{BE}}(z \rightarrow-\infty)$ and $T_{-}=T_{\mathrm{BE}}(z \rightarrow \infty)$, where $T_{\mathrm{BE}}(z)=$ $T_{+}\left(1+\delta \tau_{\mathrm{bg}}(z)\right)$ is the local temperature calculated with the Boltzmann equation. The first condition is always satisfied since we impose the boundary condition $\delta \tau_{\text {bg }}(-\infty)=0$, but we fail to recover the second one due to the different approximations made in the two methods. The discrepancy becomes larger as $v_{w}$ approaches the Jouguet velocity $\xi_{J}$, where $T_{+}$increases compared to $T_{-} \approx$ $T_{n}$ [see Fig. 6(b)]. On the other hand, $\delta \tau_{\text {bg }}$ does not change significantly in the same region. Hence, we observe an error in the temperature of order $\Delta T=T_{-}-T_{\mathrm{BE}}(\infty) \approx T_{-}-T_{+}$. Since the temperature is not accurate in the broken phase, the Higgs EOM is not automatically satisfied asymptotically. To solve that problem, we shift the actual Higgs VEV $h_{-}$ evaluated in the broken phase by an amount $-\Delta h$, so that the adjusted VEV $h_{0}=h_{-}-\Delta h$ asymptotically solves the EOM [see Eq. (24)]. This gives an additional source of uncertainty for $v_{w}$ and $L_{h}$.

We estimate the errors induced on $v_{w}$ and $L_{h}$ by $\Delta T$ and $\Delta h$, assuming they are small enough to justify keeping just the first-order terms. Assuming that $v_{w}$ is completely 


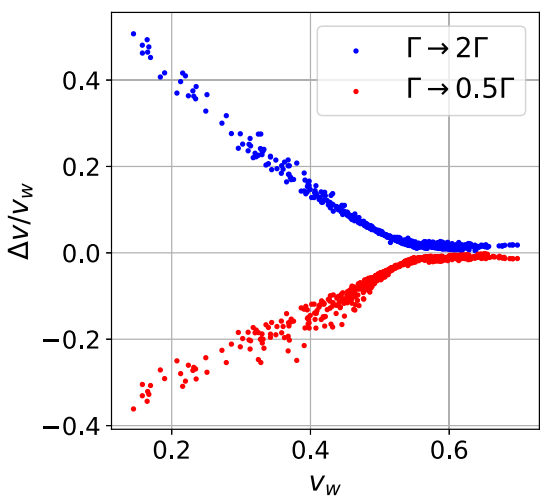

(a)

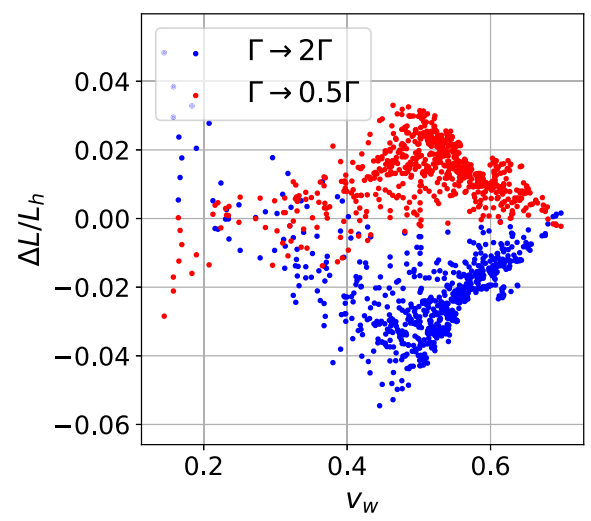

(b)

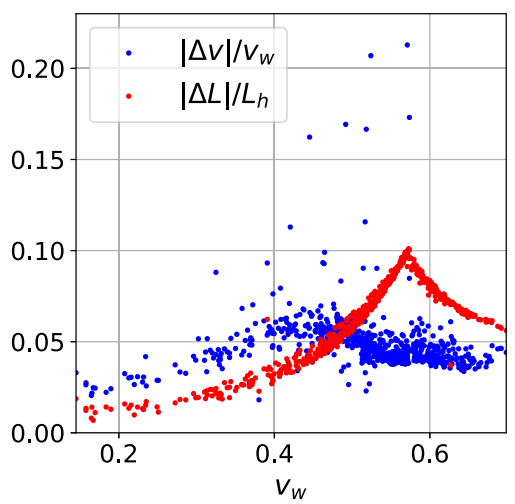

(c)

FIG. 10. (a) and (b): Relative changes $\Delta v / v_{w}$ and $\Delta L / L_{h}$ in the wall velocities and widths obtained by substituting $\Gamma \rightarrow 2 \Gamma$ or $\Gamma / 2$, respectively. (c): Absolute error on $v_{w}$ and $L_{h}$ due to the discrepancy between the temperatures computed with the Boltzmann equation and the conservation of the energy-momentum tensor [see Eq. (52)].

determined by the solution of $M_{1}=0$ and $L_{h}$ by $M_{2}=0$, the error on these solutions can be obtained by expanding around the estimated values. For example, the error in the wall velocity is estimated by

$$
\begin{aligned}
0= & M_{1}\left(v_{w}+\Delta v, h_{0}+\Delta h, T(z)+\Delta T(z)\right) \\
\approx & M_{1}\left(v_{w}, h_{0}, T(z)\right)+\frac{\partial M_{1}}{\partial v_{w}} \Delta v+\int d z \frac{\delta M_{1}}{\delta T(z)} \Delta T(z) \\
& +\Delta_{h} M_{1}
\end{aligned}
$$

where $\Delta_{h} M_{1}=M_{1}\left(v_{w}, h_{0}+\Delta h, T\right)-M_{1}\left(v_{w}, h_{0}, T\right)$, and we integrate over the temperature variation because $M_{1}$ is a functional of $T(z)$. Since $v_{w}$ is the solution of $M_{1}\left(v_{w}, h_{0}, T(z)\right)=0$, the absolute errors on $v_{w}$ and $L_{h}$ are estimated as

$$
\begin{aligned}
& |\Delta v| \approx\left(\left|\Delta_{T} M_{1}\right|+\left|\Delta_{h} M_{1}\right|\right)\left|\frac{\partial M_{1}}{\partial v_{w}}\right|^{-1}, \\
& |\Delta L| \approx\left(\left|\Delta_{T} M_{2}\right|+\left|\Delta_{h} M_{2}\right|\right)\left|\frac{\partial M_{2}}{\partial L}\right|^{-1},
\end{aligned}
$$

where $\Delta_{T} M_{i}=\int d z\left(\delta M_{i} / \delta T(z)\right) \Delta T(z)$. Notice that Eq. (49) overestimates the errors since $\Delta_{T} M_{i}$ and $\Delta_{h} M_{i}$ have opposite signs. From Eqs. (22), (25), and (26), one can see that the functional derivative $\delta M_{i} / \delta T(z)$ can be approximated by $\frac{d}{d T}\left(\partial V_{\text {eff }} / \partial h\right)$, so that

$$
\Delta_{T} M_{i} \approx \int d z \frac{d}{d T}\left(\frac{\partial V_{\mathrm{eff}}}{\partial h}\right) F_{i}(z) \Delta T(z)
$$

where $F_{1}=h^{\prime}$ and $F_{2}=h^{\prime}\left(2 h-h_{0}\right)$. We can simplify this integral with the approximation $\Delta T(z) \approx$ $\left(T_{-}-T_{+}\right)\left[1+\tanh \left(z / L_{h}\right)\right] / 2$. Furthermore, we approximate $\frac{d}{d T}\left(\frac{\partial V}{\partial h}\right)$ as being constant and half of its maximal value, occurring near $z=0$. Then

$$
\left.\Delta_{T} M_{i} \approx \frac{1}{2}\left(T_{-}-T_{+}\right) C_{i} \frac{d}{d T}\left(\frac{\partial V_{\mathrm{eff}}}{\partial h}\right)\right|_{z=0}
$$

where $C_{1}=\int d z F_{1}(z)\left[1+\tanh \left(z / L_{h}\right)\right] / 2=h_{0} / 2$ and $C_{2}=h_{0}^{2} / 6$. Substituting this expression in Eq. (49), we finally obtain that the errors on $v_{w}$ and $L_{h}$ are given by

$$
\begin{aligned}
|\Delta v| \approx & \left\{\left|\frac{1}{4}\left(T_{-}-T_{+}\right) h_{0} \frac{d}{d T}\left(\frac{\partial V_{\mathrm{eff}}}{\partial h}\right)\right|_{z=0}+\left|\Delta_{h} M_{1}\right|\right\} \\
& \times\left|\frac{\partial M_{1}}{\partial v_{w}}\right|^{-1}, \\
|\Delta L| \approx & \left\{\left|\frac{1}{12}\left(T_{-}-T_{+}\right) h_{0}^{2} \frac{d}{d T}\left(\frac{\partial V_{\mathrm{eff}}}{\partial h}\right)\right|_{z=0}+\left|\Delta_{h} M_{2}\right|\right\} \\
& \times\left|\frac{\partial M_{2}}{\partial L_{h}}\right|^{-1}
\end{aligned}
$$

The relative errors are presented in Fig. 10(c) for the scan with $\lambda_{s}=1$ and $\Lambda=540 \mathrm{GeV}$. The error on $v_{w}$ is below $7 \%$ for $97 \%$ of the models and exhibits no strong correlation with $v_{w}$. This happens because $\Delta T=T_{-}-$ $T_{+}$and $d M_{1} / d v_{w}$ are roughly proportional (see Fig. 6) and, therefore, cancel each others' contributions. The relative error on $L_{h}$ is small at low velocity (or large $L_{h}$ ) but becomes more significant near the speed of sound, however, without ever exceeding $10 \%$.

\section{E. Comparison of the GW signal with previous studies}

We end this section with a brief comparison with recent studies of the GW produced during a first-order electroweak phase transition. With the prospect of the upcoming LISA experiment, numerous forecasts of the GW spectrum have been made for various extensions of the Standard Model [48,51,125-127]. Most of these find regions of model parameter space that would produce detectable 
GWs. Here we focus on studies of the singlet scalar extensions [34,44,45,49,128,129].

Our results agree qualitatively with the conclusions of previous work, in the prediction of GWs detectable by LISA, DECIGO, and BBO. However, there are distinctions stemming from differences in methodology. To compute the GW contribution from the sound waves, previous authors used the numerical fit presented in Ref. [6], while we used the updated formulas of Refs. [67,68]. This leads to a smaller peak frequency, decreasing the number of detectable models. Reference [6] also does not include the factor $1-\left(1+2 H R / \sqrt{K_{\mathrm{sW}}}\right)^{-1 / 2}$ in the GW amplitude (see Appendix C). We find that this factor is generally quite small (of order $10^{-3}-10^{-2}$ for deflagrations and $10^{-2}-$ $10^{-1}$ for detonations); hence the predicted GW signals are considerably reduced.

Another significant difference arises from our determination of the wall velocity, which was treated as a free parameter in previous work, whereas we have computed it from the microphysics. The GW spectrum, and hence signal-to-noise ratio, and ultimately the detectability are strongly dependent on the wall speed. For example, Ref. [128] assumed $v_{w}=0.95$ for all models, which considerably enhanced GW production and led to more optimistic predictions. Moreover, using a fixed value for $v_{w}$ hides the discontinuous transition between the deflagration and detonation solutions shown in Fig. 8.

\section{CONCLUSION}

In this work we have taken a first step toward making complete predictions for baryogenesis and gravity waves from a first-order electroweak phase transition, starting from a renormalizable Lagrangian that gives rise to the effective operator needed for $C P$ violation. This is in contrast to previous studies in which quantities like the bubble wall velocity or thickness were treated as free parameters, instead of being derived from the microphysical input parameters as we have done here. This is a necessary step for properly assessing the chances of having successful EWBG and potentially observable GWs, since the two observables are correlated in a nontrivial way, when they are both computed from first principles.

We have incorporated improved fluid equations, both for the $C P$-even perturbations that determine the friction acting on the bubble wall [66], and for the $C P$-odd ones that are necessary for baryogenesis [65], that can properly account for wall speeds close to the sound barrier. Earlier versions of these equations were singular at the sound speed, making reliable predictions impossible for fast-moving walls. Contrary to previous lore, we find that EWBG can be more efficient for faster walls, due in part to the tendency for fast walls to be thinner.

The $Z_{2}$-symmetric singlet model with vectorlike top partners, analyzed in this work, was chosen for its simplicity, but the methods we used can be applied to other particle physics models that could enhance the EWPT. For example, singlets with no $Z_{2}$ symmetry have additional parameters and would, thus, be likely to have more freedom to simultaneously yield large GW production and sufficient baryogenesis. It would be interesting to identify other UVcompleted models with these properties. A limitation we identified with the $Z_{2}$-symmetric model is that for the large values of the $\eta_{2}$ coupling that are desired for EWBG, the singlet self-coupling is rapidly driven toward zero by renormalization group running, above the top partner threshold.

For future work, some improvements could be made to the analysis presented here. The wall velocity might be more accurately determined at low $v_{w}$ by using collision rates for the fluid perturbation equations beyond leading$\log$ accuracy $[60,130]$ and by including the singlet and Higgs out-of-equilibrium (friction) contributions [56]. Another limitation is that the current state-of-the-art for predicting the GW spectrum is subject to large systematic uncertainties for wall velocities close to the speed of sound [7]. Since a large fraction of deflagration transitions have $0.5 \lesssim v_{w} \lesssim \xi_{J}$, our analysis of the GW production could greatly benefit from more accurate fits in that range of wall speeds.

\section{ACKNOWLEDGMENTS}

We thank T. Flacke, H. Guo, M. Lewicki, K. Schmitz, G. Servant, and K.-P. Xie for helpful correspondence. The work of J.C. and B. L. was supported by the Natural Sciences and Engineering Research Council (Canada). The work of B. L. was also supported by the Fonds de recherche Nature et technologies (Québec). The work of K. K. was supported by the Academy of Finland Grant No. 31831.

\section{APPENDIX A: EFFECTIVE POTENTIAL}

We describe here the full effective potential used to describe the phase transition in the $Z_{2}$-symmetric singlet model. It takes the general form

$$
\begin{aligned}
V_{\text {eff }}(h, s, T)= & V_{\text {tree }}(h, s)+V_{\mathrm{CW}}(h, s, T) \\
& +V_{T}(h, s, T)+\delta V(h, s) .
\end{aligned}
$$

Note that $V_{\text {tree }}$ is the scalar degrees of freedom's tree-level potential obtained in the unitary gauge by setting in Eq. (1) $H \rightarrow h / \sqrt{2}$ and by omitting the $V_{\mathrm{BG}}$ term:

$V_{\text {tree }}(h, s)=\frac{\mu_{h}^{2}}{2} h^{2}+\frac{\lambda_{h}}{4} h^{4}+\frac{\lambda_{h s}}{4} h^{2} s^{2}+\frac{\mu_{s}^{2}}{2} s^{2}+\frac{\lambda_{s}}{4} s^{4}$.

Note that $V_{\mathrm{CW}}$ is the Coleman-Weinberg potential in the $\overline{\mathrm{MS}}$ renormalization scheme that incorporates the vacuum one-loop corrections, and $V_{T}$ is the thermal potential: 


$$
\begin{aligned}
V_{\mathrm{CW}}(h, s, T) & =\frac{1}{64 \pi^{2}} \sum_{i=W, Z, \gamma_{L}, 1,2, \chi, t} n_{i} \tilde{\mathcal{M}}_{i}^{4}(h, s, T)\left[\log \frac{\tilde{\mathcal{M}}_{i}^{2}(h, s, T)}{\mu^{2}}-C_{i}\right] \\
V_{T}(h, s, T) & =\sum_{i=W, Z, \gamma_{L}, 1,2, \chi, t} \frac{n_{i} T^{4}}{2 \pi^{2}} \int_{0}^{\infty} d y y^{2} \log \left[1 \pm e^{-\sqrt{y^{2}+\mathcal{M}_{i}^{2}(h, s, T) / T^{2}}}\right]-\frac{\tilde{g} \pi^{2} T^{4}}{90},
\end{aligned}
$$

where the sums go over all the massive particles, including the thermal mass. Here, we include the contribution from the $W$ and $Z$ gauge bosons, the photon's longitudinal polarization $\gamma_{L}$, the Goldstone bosons $\chi$, the top quark and the eigenvalues of the mass matrix of the Higgs boson, and singlet scalar $m_{1}$ and $m_{2}$. We impose the renormalization energy scale as $\mu=v$, where $v=246 \mathrm{GeV}$ is the Higgs vacuum expectation value. The \pm in the thermal integral is + for fermion and - for bosons, and $\tilde{g}=$ $\sum_{B} N_{B}+\frac{7}{8} \sum_{F} N_{F}=85.25$ with the sums running over all the lighter degrees of freedom not included in the first term of $V_{T}$. The $C_{i}$ 's are constants given by

$$
C_{1,2, \chi, t}=3 / 2 \quad \text { and } \quad C_{W, Z, \gamma_{L}}=5 / 6 \text {, }
$$

and the $n_{i}$ 's are the particle's number of degrees of freedom:

$n_{W_{T}}=4, \quad n_{W_{L}}=n_{Z_{T}}=2, \quad n_{Z_{L}}=n_{\gamma_{L}}=1$,

$n_{1,2}=1, \quad n_{\chi}=3, \quad n_{t}=-12$.

We adopt the method developed by Parwani [73] to resum the Matsubara zero modes for the bosonic degrees of freedom. It consists of replacing the bosons' vacuum mass $m_{i}^{2}(h, s)$ by the thermal-corrected one $\mathcal{M}_{i}^{2}(h, s, T)=$ $m_{i}^{2}(h, s)+\Pi_{i}(T)$, with the self-energy given by

$$
\begin{aligned}
\Pi_{s}(T) & =\left(\frac{1}{4} \lambda_{s}+\frac{1}{6} \lambda_{s h}\right) T^{2}, \\
\Pi_{h}(T) & =\Pi_{\chi}(T)=\left[\frac{1}{16}\left(3 g_{1}^{2}+g_{2}^{2}\right)+\frac{1}{2} \lambda_{h}+\frac{1}{4} y_{t}^{2}+\frac{1}{24} \lambda_{h s}\right] T^{2}, \\
\Pi_{W_{L}}(T) & =\frac{11}{6} g_{1}^{2} T^{2}, \\
\Pi_{W_{T}}(T) & =\Pi_{Z_{T}}(T)=\Pi_{\gamma_{T}}(T)=0 .
\end{aligned}
$$

The thermal masses for the longitudinal mode of the photon and $Z$ boson are

$\mathcal{M}_{Z_{L}}^{2}(s, h, T)=\frac{1}{2}\left[m_{Z}^{2}(s, h)+\frac{11}{6} \frac{g_{1}^{2}}{\cos ^{2} \theta_{w}} T^{2}+\Delta(s, h, T)\right]$

and

$\mathcal{M}_{\gamma_{L}}^{2}(s, h, T)=\frac{1}{2}\left[m_{Z}^{2}(s, h)+\frac{11}{6} \frac{g_{1}^{2}}{\cos ^{2} \theta_{w}} T^{2}-\Delta(s, h, T)\right]$,

with

$$
\Delta(s, h, T)=\left[m_{Z}^{4}(s, h)+\frac{11}{3} \frac{g_{1}^{2} \cos ^{2} 2 \theta_{w}}{\cos ^{2} \theta_{w}}\left(m_{Z}^{2}(s, h)+\frac{11}{12} \frac{g_{1}^{2}}{\cos ^{2} \theta_{w}} T^{2}\right) T^{2}\right]^{1 / 2} .
$$

At low temperature $\left(m_{i}^{2} / T^{2} \gg 1\right)$, one would expect all the thermal effects to be Boltzmann suppressed since the species $i$ becomes essentially absent from the plasma. In the Parwani method, this is manifestly the case for $V_{T}$ since the thermal integrals decay exponentially in the limit $\mathcal{M}_{i}^{2} / T^{2} \approx m_{i}^{2} / T^{2} \gg 1$. In this way Parwani resummation is better than the formally more correct ring resummation, where only the zero modes are dressed [74-76], but where the ring correction to $V_{T}$ is not correctly suppressed at low temperatures. However, in the same limit, $V_{\mathrm{CW}}$ would depend quadratically on $T$ if we used the thermal masses defined above. This would spoil the potential's low- $T$ behavior. A consistent treatment of this problem would require summing thermal corrections beyond the one-loop order, keeping track of a correct renormalization. This could be done using 2PI techniques [131,132], but goes beyond the scope of this work. Instead, we impose the correct Boltzmann suppression by hand, defining a regulated thermal mass, ${ }^{12} \tilde{\mathcal{M}}_{i}^{2}=m_{i}^{2}+R\left(m_{i}^{2} / T^{2}\right) \Pi_{i}$, that should only be used in $V_{\mathrm{CW}}$. Note that $R(x)$ is a regulator chosen to recover the right behavior in the low and high- $T$ limit. A well-motivated choice is $R(x) \equiv I_{0}(x) / I_{0}(0)$, where $I_{0}(x)=2 d J(x) / d x$ and $J(x)$ is the thermal $J$-integral appearing in the sum in Eq. (A3). In the Maxwell-Boltzmann approximation, this becomes

$$
R(x)=\sqrt{x} K_{1}(\sqrt{x})
$$

where $K_{1}$ is the modified Bessel function of the second kind. We have checked numerically that the precise choice

\footnotetext{
${ }^{12}$ For the photon and $Z$ boson's longitudinal mode, we define $\Pi_{i}=\mathcal{M}_{i}^{2}-m_{i}^{2}$, which should reproduce the desired behavior.
} 
of regulator is not important, as long as it provides a correct interpolation to the high-temperature limit and the right Boltzmann suppression at low temperatures.

The last term of Eq. (A1) contains the following counterterms:

$$
\delta V(h, s)=A h^{2}+B h^{4}+C s^{2}+D,
$$

which are fixed by requiring the renormalization conditions

$$
\begin{gathered}
0=\left.\frac{\partial V_{\text {eff }}}{\partial h}\right|_{h=v, s=0, T=0} \\
m_{h}^{2}=\left.\frac{\partial^{2} V_{\text {eff }}}{\partial h^{2}}\right|_{h=v, s=0, T=0} \\
m_{s}^{2}=\left.\frac{\partial^{2} V_{\text {eff }}}{\partial s^{2}}\right|_{h=v, s=0, T=0} \\
0=\left.V_{\text {eff }}\right|_{h=v, s=0, T=0} .
\end{gathered}
$$

While the use of the resummed one-loop potential is a clear improvement over the leading thermal-mass-corrected approximation, one should keep in mind that higher loop corrections and even nonperturbative physics may be relevant, in particular, for very strong transitions [133-135].

\section{APPENDIX B: RELATIVISTIC FLUID EQUATION}

We here calculate the hydrodynamical properties of the plasma close to the wall using the method described in Ref. [98]. The quantities of interest are the temperatures $T_{ \pm}$ and the velocities of the plasma measured in the wall frame $v_{ \pm}$. The subscript + and - indicate that the quantity is measured in front or behind the wall, respectively.

By integrating the conservation of the energy-momentum tensor equation across the wall, one can show that the quantities $T_{ \pm}$and $v_{ \pm}$are related by the equations

$$
\begin{aligned}
v_{+} v_{-} & =\frac{1-\left(1-3 \alpha_{+}\right) r}{3-3\left(1+\alpha_{+}\right) r}, \\
\frac{v_{+}}{v_{-}} & =\frac{3+\left(1-3 \alpha_{+}\right) r}{1+3\left(1+\alpha_{+}\right) r},
\end{aligned}
$$

where $\alpha_{+}$and $r$ are defined as

$$
\begin{aligned}
\alpha_{+} & \equiv \frac{\epsilon_{+}-\epsilon_{-}}{a_{+} T_{+}^{4}}, \\
r & \equiv \frac{a_{+} T_{+}^{4}}{a_{-} T_{-}^{4}}, \\
a_{ \pm} & \equiv-\left.\frac{3}{4 T_{ \pm}^{3}} \frac{\partial V_{\mathrm{eff}}}{\partial T}\right|_{ \pm}, \\
\epsilon_{ \pm} & \left.\equiv\left(-\frac{T_{ \pm}}{4} \frac{\partial V_{\mathrm{eff}}}{\partial T}+V_{\mathrm{eff}}\right)\right|_{ \pm} .
\end{aligned}
$$

These quantities are often approximated by the so-called bag equation of state, which is given in Ref. [98]. This approximation is expected to hold when the masses of the plasma's degrees of freedom are very different from $T$, which is not necessarily true in the broken phase. Therefore, we keep the full relations (B2) in our calculations.

Subsonic walls always come with a shock wave in front of the phase transition front. The Eqs. (B1) can be used to relate $T_{ \pm}$and $v_{ \pm}$at the wall and the shock wave, but we need to understand how the temperature and fluid velocity evolve between these two regions. Assuming a spherical bubble and a thin wall, one can derive from the conservation of the energymomentum tensor the following differential equations:

$$
\begin{aligned}
2 \frac{v}{\xi} & =\gamma^{2}(1-v \xi)\left(\frac{\mu^{2}}{c_{s}^{2}}-1\right) \partial_{\xi} v, \\
\partial_{\xi} T & =T \gamma^{2} \mu \partial_{\xi} v,
\end{aligned}
$$

where $v$ is the fluid velocity in the frame of the bubble's center, and $\xi=r / t$ is the independent variable, with $r$ the distance from the bubble center and $t$ the time since the bubble nucleation. With that choice of coordinates, the wall is positioned at $\xi=v_{w}$. Note that $\mu$ is the Lorentz-transformed fluid velocity

$$
\mu(\xi, v)=\frac{\xi-v}{1-\xi v},
$$

and $c_{s}$ is the speed of sound in the plasma

$$
c_{s}^{2}=\frac{\partial V_{\text {eff }} / \partial T}{T \partial^{2} V_{\text {eff }} / \partial T^{2}} \approx \frac{1}{3} .
$$

The last approximation is valid for relativistic fluids, which models well the unbroken phase. In the broken phase, the particles get a mass that can be of the same order as the temperature, and it causes the speed of sound to become slightly smaller.

One can find three different types of solutions for the fluid's velocity profile: deflagration walls $\left(v_{w}<c_{s}^{-}\right)$have a shock wave propagating in front of the wall, detonation walls $\left(v_{w}>\xi_{J}\right)$ have a rarefaction wave behind it, and hybrid walls $\left(c_{s}^{-}<v_{w}<\xi_{J}\right)$ have both shock and rarefaction waves. Note that $\xi_{J}$ is the model-dependent Jouguet velocity, which is defined as the smallest velocity a detonation solution can have. Each type of wall have different boundary conditions that determine the characteristics of the solution. Detonation walls are supersonic solutions where the fluid in front of the wall is unperturbed. Therefore, it satisfies the boundary conditions $v_{+}=v_{w}$ and $T_{+}=T_{n}$. For that type of solution, Eqs. (B1) can be solved directly for $v_{-}$and $T_{-}$.

Subsonic walls always have a deflagration solution with a shock wave at a position $\xi_{s h}$ that solves the equation $v_{s h}^{-} \xi_{s h}=\left(c_{s}^{+}\right)^{2}$, where $v_{s h}^{-}$is the fluid's velocity just behind the shock wave measured in the shock wave's frame. 
It satisfies the boundary conditions $v_{-}=v_{w}$ and $T_{s h}^{+}=T_{n}$. Because these boundary conditions are given at two different points, the solution of this system can be somewhat more involved than for the detonation case. Indeed, one has to use a shooting method, which consists of choosing an arbitrary value for $T_{-}$, solving Eqs. (B1) for $T_{+}$and $v_{+}$, integrating Eqs. (B3) with the initial values $T\left(v_{w}\right)=T_{+}$and $v\left(v_{w}\right)=\mu\left(v_{w}, v_{+}\right)$until the equation $\mu(\xi, v(\xi)) \xi=\left(c_{s}^{+}\right)^{2}$ gets satisfied. One can then restart this procedure with a different value of $T_{-}$until the Eqs. (B1) are satisfied at the shock wave. Hybrid walls satisfy $v_{+}<c_{s}^{-}<v_{w}$, and they have the boundary conditions $v_{-}=c_{s}^{-}$and $T_{s h}^{+}=T_{n}$, which make them very similar to the deflagration walls.

\section{APPENDIX C: GRAVITATIONAL WAVE PRODUCTION}

For the convenience of the reader, we here reproduce the formulae from Refs. $[7,67,68,98,100]$ that determine the GW spectrum from sound waves and turbulence in a firstorder phase transition. The spectrum is $[67,68]$

$$
\Omega_{\mathrm{sw}}(f)=8.83 \times 10^{-7} K_{\mathrm{sw}}^{2}\left(\frac{H R}{c_{s}}\right)\left(1-\left(1+\frac{2 H R}{\sqrt{K_{\mathrm{sw}}}}\right)^{-1 / 2}\right)\left(\frac{100}{g_{*}}\right)^{1 / 3} S_{\mathrm{sw}}(f),
$$

where $K_{\mathrm{sw}}=\kappa_{\mathrm{sw}} \alpha /(1+\alpha)$, with $\kappa_{\mathrm{sw}}$ the efficiency coefficient of the sound wave. As previously stated, we assume that all the walls have nonrunaway solutions and that the contribution from turbulence is negligible; hence we set $\Omega_{\mathrm{sw}}=\Omega_{\phi}(f)=0$. The function parametrizing the shape of the GW spectrum is

$$
S_{\mathrm{sw}}(f)=\left(\frac{f}{f_{\mathrm{sw}}}\right)^{3}\left(\frac{7}{4+3\left(f / f_{\mathrm{sw}}\right)^{2}}\right)^{\frac{7}{2}},
$$

and the peak frequency $f_{\mathrm{sw}}$ is

$$
f_{\mathrm{sw}}=2.6 \times 10^{-5} \mathrm{~Hz}\left(\frac{1}{H R}\right)\left(\frac{T_{n}}{100 \mathrm{GeV}}\right)\left(\frac{g_{*}}{100}\right)^{\frac{1}{6}} .
$$

Numerical fits for the efficiency coefficient $\kappa_{\mathrm{sw}}$ (the fractions of the available vacuum energy that go into kinetic energy) were presented in [98]. For nonrunaway walls, these fits depend on the wall velocity and are given by

$$
\kappa_{\mathrm{SW}}= \begin{cases}\frac{c_{s}^{11 / 5} \kappa_{a} \kappa_{b}}{\left(c_{s}^{11 / 5}-v_{w}^{11 / 5}\right) \kappa_{b}+v_{w} c_{s}^{6 / 5} \kappa_{a}}, & v_{w} \lesssim c_{s} \\ \kappa_{b}+\left(v_{w}-c_{S}\right) \delta \kappa+\frac{\left(v_{w}-c_{s}\right)^{3}}{\left(\xi_{J}-c_{s}\right)^{3}}\left[\kappa_{c}-\kappa_{b}-\left(\xi_{J}-c_{S}\right) \delta \kappa\right], & c_{s}<v_{w}<\xi_{J} \\ \frac{\left(\xi_{J}-1\right)^{3} \xi_{J}^{5 / 2} v_{w}^{-5 / 2} \kappa_{c} \kappa_{d}}{\left[\left(\xi_{J}-1\right)^{3}-\left(v_{w}-1\right)^{3}\right] \xi_{J}^{5 / 2} \kappa_{c}+\left(v_{w}-1\right)^{3} \kappa_{d}}, & v_{w} \gtrsim \xi_{J},\end{cases}
$$

where $c_{s}=1 / \sqrt{3}$ is the sound velocity, and the different parameters are given by

$$
\begin{aligned}
& \xi_{J}=\frac{\sqrt{2 \alpha / 3+\alpha^{2}}+c_{s}}{1+\alpha} \quad \delta \kappa=-0.9 \log \frac{\sqrt{\alpha}}{1+\sqrt{\alpha}} \\
& \kappa_{a}=\frac{6.9 v_{w}^{6 / 5} \alpha}{1.36-0.037 \sqrt{\alpha}+\alpha} \kappa_{b}=\frac{\alpha^{2 / 5}}{0.017+(0.997+\alpha)^{2 / 5}} \\
& \kappa_{c}=\frac{\sqrt{\alpha}}{0.135+\sqrt{0.98+\alpha}} \kappa_{d}=\frac{\alpha}{0.73+0.083 \sqrt{\alpha}+\alpha} .
\end{aligned}
$$

We caution that while these fits, when used as input for a signal-to-noise ratio estimate, are useful to get an overall estimate for the GW signal in a given model, their precise predictions should be interpreted with care. The fit for the sound wave production is reliable for relatively weak transitions $\alpha<0.1$, which is the range where most of our models fall. For stronger transitions the fit can overestimate the GW signal by as much as a factor of a thousand (strong deflagrations) [136]. In addition to the strength of the transition, fit parameters have also been shown to be sensitive to the shape of the effective potential [137] and the wall velocity [7,68]. As explained in Ref. [7], Eqs. (C1)-(C3) are not expected to be accurate for $0.5 \lesssim v_{w} \lesssim \xi_{J}$, which includes a large fraction of the deflagration models found in this work. Thus, pending improvements in the theoretical predictions for $\mathrm{GW}$ spectra in this range of wall speeds, the results should not be regarded as conclusive. 
[1] A. I. Bochkarev, S. V. Kuzmin, and M. E. Shaposhnikov, Electroweak baryogenesis and the Higgs boson mass problem, Phys. Lett. B 244, 275 (1990).

[2] A. G. Cohen, D. B. Kaplan, and A. E. Nelson, Weak scale baryogenesis, Phys. Lett. B 245, 561 (1990).

[3] A. G. Cohen, D. B. Kaplan, and A. E. Nelson, Baryogenesis at the weak phase transition, Nucl. Phys. B349, 727 (1991).

[4] N. Turok and J. Zadrozny, Electroweak baryogenesis in the two doublet model, Nucl. Phys. B358, 471 (1991).

[5] A. J. Long, A. Tesi, and L.-T. Wang, Baryogenesis at a lepton-number-breaking phase transition, J. High Energy Phys. 10 (2017) 095.

[6] C. Caprini et al., Science with the space-based interferometer eLISA. II: Gravitational waves from cosmological phase transitions, J. Cosmol. Astropart. Phys. 04 (2016) 001.

[7] C. Caprini et al., Detecting gravitational waves from cosmological phase transitions with LISA: An update, J. Cosmol. Astropart. Phys. 03 (2020) 024.

[8] J. Crowder and N. J. Cornish, Beyond LISA: Exploring future gravitational wave missions, Phys. Rev. D 72, 083005 (2005).

[9] N. Seto, S. Kawamura, and T. Nakamura, Possibility of Direct Measurement of the Acceleration of the Universe Using 0.1-Hz Band Laser Interferometer Gravitational Wave Antenna in Space, Phys. Rev. Lett. 87, 221103 (2001).

[10] S. Sato et al., The status of DECIGO, J. Phys. Conf. Ser. 840, 012010 (2017).

[11] Y. A. El-Neaj et al. (AEDGE Collaboration), AEDGE: Atomic experiment for dark matter and gravity exploration in space, Eur. Phys. J. Quantum Technol. 7, 6 (2020).

[12] K. Kajantie, M. Laine, K. Rummukainen, and M. E. Shaposhnikov, A Nonperturbative analysis of the finite $\mathrm{T}$ phase transition in $\mathrm{SU}(2) \times \mathrm{U}(1)$ electroweak theory, Nucl. Phys. B493, 413 (1997).

[13] K. Kajantie, M. Laine, K. Rummukainen, and M. E. Shaposhnikov, Is There a Hot Electroweak Phase Transition at $\mathrm{m}(\mathrm{H})$ Larger or Equal to $\mathrm{m}(\mathrm{W})$ ?, Phys. Rev. Lett. 77, 2887 (1996).

[14] G. W. Anderson and L. J. Hall, The electroweak phase transition and baryogenesis, Phys. Rev. D 45, 2685 (1992).

[15] J. McDonald, Electroweak baryogenesis and dark matter via a gauge singlet scalar, Phys. Lett. B 323, 339 (1994).

[16] J. Choi and R. R. Volkas, Real Higgs singlet and the electroweak phase transition in the standard model, Phys. Lett. B 317, 385 (1993).

[17] J. R. Espinosa and M. Quiros, Novel effects in electroweak breaking from a hidden sector, Phys. Rev. D 76, 076004 (2007).

[18] S. Profumo, M. J. Ramsey-Musolf, and G. Shaughnessy, Singlet Higgs phenomenology and the electroweak phase transition, J. High Energy Phys. 08 (2007) 010.

[19] J. R. Espinosa, T. Konstandin, and F. Riva, Strong electroweak phase transitions in the standard model with a singlet, Nucl. Phys. B854, 592 (2012).

[20] J. M. Cline, K. Kainulainen, P. Scott, and C. Weniger, Update on scalar singlet dark matter, Phys. Rev. D 88, 055025 (2013); Erratum, Phys. Rev. D 92, 039906 (2015).
[21] P. H. Damgaard, A. Haarr, D. O'Connell, and A. Tranberg, Effective field theory and electroweak baryogenesis in the singlet-extended standard model, J. High Energy Phys. 02 (2016) 107.

[22] G. Kurup and M. Perelstein, Dynamics of electroweak phase transition in singlet-scalar extension of the standard model, Phys. Rev. D 96, 015036 (2017).

[23] C.-W. Chiang, Y.-T. Li, and E. Senaha, Revisiting electroweak phase transition in the standard model with a real singlet scalar, Phys. Lett. B 789, 154 (2019).

[24] S. W. Ham, Y. S. Jeong, and S. K. Oh, Electroweak phase transition in an extension of the standard model with a real Higgs singlet, J. Phys. G 31, 857 (2005).

[25] A. Noble and M. Perelstein, Higgs self-coupling as a probe of electroweak phase transition, Phys. Rev. D 78, 063518 (2008).

[26] D. Curtin, P. Meade, and C.-T. Yu, Testing electroweak baryogenesis with future colliders, J. High Energy Phys. 11 (2014) 127.

[27] S. Profumo, M. J. Ramsey-Musolf, C. L. Wainwright, and P. Winslow, Singlet-catalyzed electroweak phase transitions and precision Higgs boson studies, Phys. Rev. D 91, 035018 (2015).

[28] A. V. Kotwal, M. J. Ramsey-Musolf, J. M. No, and P. Winslow, Singlet-catalyzed electroweak phase transitions in the $100 \mathrm{TeV}$ frontier, Phys. Rev. D 94, 035022 (2016).

[29] P. Huang, A. J. Long, and L.-T. Wang, Probing the electroweak phase transition with Higgs factories and gravitational waves, Phys. Rev. D 94, 075008 (2016).

[30] C.-Y. Chen, J. Kozaczuk, and I. M. Lewis, Non-resonant collider signatures of a singlet-driven electroweak phase transition, J. High Energy Phys. 08 (2017) 096.

[31] J. H. Kim and I. M. Lewis, Loop induced single top partner production and decay at the LHC, J. High Energy Phys. 05 (2018) 095.

[32] K. Hashino, R. Jinno, M. Kakizaki, S. Kanemura, T. Takahashi, and M. Takimoto, Selecting models of firstorder phase transitions using the synergy between collider and gravitational-wave experiments, Phys. Rev. D 99, 075011 (2019).

[33] M. J. Ramsey-Musolf, The electroweak phase transition: A collider target, J. High Energy Phys. 09 (2020) 179.

[34] K.-P. Xie, Lepton-mediated electroweak baryogenesis, gravitational waves and the $4 \tau$ final state at the collider, J. High Energy Phys. 02 (2021) 090.

[35] S. Adhikari, I. M. Lewis, and M. Sullivan, Beyond the Standard Model effective field theory: The singlet extended Standard Model, Phys. Rev. D 103, 075027 (2021).

[36] S. Das, P. J. Fox, A. Kumar, and N. Weiner, The dark side of the electroweak phase transition, J. High Energy Phys. 11 (2010) 108.

[37] A. Ashoorioon and T. Konstandin, Strong electroweak phase transitions without collider traces, J. High Energy Phys. 07 (2009) 086.

[38] M. Jiang, L. Bian, W. Huang, and J. Shu, Impact of a complex singlet: Electroweak baryogenesis and dark matter, Phys. Rev. D 93, 065032 (2016).

[39] M. Kakizaki, S. Kanemura, and T. Matsui, Gravitational waves as a probe of extended scalar sectors with the first 
order electroweak phase transition, Phys. Rev. D 92, 115007 (2015).

[40] K. Hashino, M. Kakizaki, S. Kanemura, and T. Matsui, Synergy between measurements of gravitational waves and the triple-Higgs coupling in probing the first-order electroweak phase transition, Phys. Rev. D 94, 015005 (2016).

[41] M. Chala, G. Nardini, and I. Sobolev, Unified explanation for dark matter and electroweak baryogenesis with direct detection and gravitational wave signatures, Phys. Rev. D 94, 055006 (2016).

[42] T. Tenkanen, K. Tuominen, and V. Vaskonen, A strong electroweak phase transition from the inflaton field, J. Cosmol. Astropart. Phys. 09 (2016) 037.

[43] K. Hashino, M. Kakizaki, S. Kanemura, P. Ko, and T. Matsui, Gravitational waves and Higgs boson couplings for exploring first order phase transition in the model with a singlet scalar field, Phys. Lett. B 766, 49 (2017).

[44] V. Vaskonen, Electroweak baryogenesis and gravitational waves from a real scalar singlet, Phys. Rev. D 95, 123515 (2017).

[45] A. Beniwal, M. Lewicki, J. D. Wells, M. White, and A. G. Williams, Gravitational wave, collider and dark matter signals from a scalar singlet electroweak baryogenesis, J. High Energy Phys. 08 (2017) 108.

[46] A. Ahriche, K. Hashino, S. Kanemura, and S. Nasri, Gravitational waves from phase transitions in models with charged singlets, Phys. Lett. B 789, 119 (2019).

[47] S. De Curtis, L. Delle Rose, and G. Panico, Composite dynamics in the early universe, J. High Energy Phys. 12 (2019) 149.

[48] A. Beniwal, M. Lewicki, M. White, and A. G. Williams, Gravitational waves and electroweak baryogenesis in a global study of the extended scalar singlet model, J. High Energy Phys. 02 (2019) 183.

[49] M. Carena, Z. Liu, and Y. Wang, Electroweak phase transition with spontaneous $\mathrm{Z}_{2}$-breaking, J. High Energy Phys. 08 (2020) 107.

[50] R. Zhou, L. Bian, and H.-K. Guo, Connecting the electroweak sphaleron with gravitational waves, Phys. Rev. D 101, 091903 (2020).

[51] J. Ellis, M. Lewicki, and V. Vaskonen, Updated predictions for gravitational waves produced in a strongly supercooled phase transition, J. Cosmol. Astropart. Phys. 11 (2020) 020 .

[52] G. D. Moore and T. Prokopec, How fast can the wall move? A study of the electroweak phase transition dynamics, Phys. Rev. D 52, 7182 (1995).

[53] G. D. Moore and T. Prokopec, Bubble Wall Velocity in a First Order Electroweak Phase Transition, Phys. Rev. Lett. 75, 777 (1995).

[54] P. John and M. G. Schmidt, Do stops slow down electroweak bubble walls?, Nucl. Phys. B598, 291 (2001); Erratum, Nucl. Phys. B648, 449 (2003).

[55] D. Bodeker and G. D. Moore, Can electroweak bubble walls run away?, J. Cosmol. Astropart. Phys. 05 (2009) 009.

[56] J. Kozaczuk, Bubble expansion and the viability of singletdriven electroweak baryogenesis, J. High Energy Phys. 10 (2015) 135.
[57] T. Konstandin and J. M. No, Hydrodynamic obstruction to bubble expansion, J. Cosmol. Astropart. Phys. 02 (2011) 008.

[58] T. Konstandin, G. Nardini, and I. Rues, From Boltzmann equations to steady wall velocities, J. Cosmol. Astropart. Phys. 09 (2014) 028.

[59] D. Bodeker and G. D. Moore, Electroweak bubble wall speed limit, J. Cosmol. Astropart. Phys. 05 (2017) 025.

[60] S. Höche, J. Kozaczuk, A. J. Long, J. Turner, and Y. Wang, Towards an all-orders calculation of the electroweak bubble wall velocity, J. Cosmol. Astropart. Phys. 03 (2021) 009.

[61] M. Barroso Mancha, T. Prokopec, and B. Swiezewska, Field-theoretic derivation of bubble-wall force, J. High Energy Phys. 01 (2021) 070.

[62] S. Balaji, M. Spannowsky, and C. Tamarit, Cosmological bubble friction in local equilibrium, J. Cosmol. Astropart. Phys. 03 (2021) 051.

[63] A. Azatov and M. Vanvlasselaer, Bubble wall velocity: Heavy physics effects, J. Cosmol. Astropart. Phys. 01 (2021) 058.

[64] A. Friedlander, I. Banta, J. M. Cline, and D. Tucker-Smith, Wall speed and shape in singlet-assisted strong electroweak phase transitions, Phys. Rev. D 103, 055020 (2021).

[65] J. M. Cline and K. Kainulainen, Electroweak baryogenesis at high bubble wall velocities, Phys. Rev. D 101, 063525 (2020).

[66] B. Laurent and J. M. Cline, Fluid equations for fastmoving electroweak bubble walls, Phys. Rev. D 102, 063516 (2020).

[67] H.-K. Guo, K. Sinha, D. Vagie, and G. White, Phase transitions in an expanding universe: Stochastic gravitational waves in standard and non-standard histories, J. Cosmol. Astropart. Phys. 01 (2021) 001.

[68] M. B. Hindmarsh, M. Lüben, J. Lumma, and M. Pauly, Phase transitions in the early universe, SciPost Phys. Lect. Notes 24, 1 (2021).

[69] W. Waltenberger, A. Lessa, and S. Kraml, Artificial protomodelling: Building precursors of a next standard model from simplified model results, J. High Energy Phys. 03 (2021) 207.

[70] J. de Vries, M. Postma, J. van de Vis, and G. White, Electroweak baryogenesis and the standard model effective field theory, J. High Energy Phys. 01 (2018) 089.

[71] M. Postma and G. White, Cosmological phase transitions: Is effective field theory just a toy?, J. High Energy Phys. 03 (2021) 280.

[72] J. M. Cline and K. Kainulainen, Electroweak baryogenesis and dark matter from a singlet Higgs, J. Cosmol. Astropart. Phys. 01 (2013) 012.

[73] R. R. Parwani, Resummation in a hot scalar field theory, Phys. Rev. D 45, 4695 (1992); , Erratum, Phys. Rev. D 48, 5965 (1993).

[74] M. Carrington, The effective potential at finite temperature in the Standard Model, Phys. Rev. D 45, 2933 (1992).

[75] P. B. Arnold and O. Espinosa, The Effective potential and first order phase transitions: Beyond leading-order, Phys. Rev. D 47, 3546 (1993); Erratum, Phys. Rev. D 50, 6662 (1994). 
[76] P. B. Arnold and O. Espinosa, The Effective potential and first order phase transitions: Beyond leading-order, Phys. Rev. D 47, 3546 (1993); Erratum, Phys. Rev. D 50, 6662 (1994).

[77] A. M. Sirunyan et al. (CMS Collaboration), Combined measurements of Higgs boson couplings in proton-proton collisions at $\sqrt{s}=13 \mathrm{TeV}$, Eur. Phys. J. C 79, 421 (2019).

[78] G. Aad et al. (ATLAS Collaboration), Combined measurements of Higgs boson production and decay using up to $80 \mathrm{fb}^{-1}$ of proton-proton collision data at $\sqrt{s}=13 \mathrm{TeV}$ collected with the ATLAS experiment, Phys. Rev. D 101, 012002 (2020).

[79] M. Aaboud et al. (ATLAS Collaboration), Combination of the Searches for Pair-Produced Vector-Like Partners of the Third-Generation Quarks at $\sqrt{s}=13 \mathrm{TeV}$ with the ATLAS Detector, Phys. Rev. Lett. 121, 211801 (2018).

[80] A. M. Sirunyan et al. (CMS Collaboration), Search for vector-like $\mathrm{T}$ and $\mathrm{B}$ quark pairs in final states with leptons at $\sqrt{s}=13 \mathrm{TeV}$, J. High Energy Phys. 08 (2018) 177.

[81] G. Brooijmans et al., Les Houches 2019 Physics at TeV Colliders: New Physics Working Group Report, arXiv: 2002.12220.

[82] G. Cacciapaglia, T. Flacke, M. Park, and M. Zhang, Exotic decays of top partners: Mind the search gap, Phys. Lett. B 798, 135015 (2019).

[83] S. Dawson and E. Furlan, A Higgs conundrum with vector fermions, Phys. Rev. D 86, 015021 (2012).

[84] M. Tanabashi et al. (Particle Data Group Collaboration), Review of particle physics, Phys. Rev. D 98, 030001 (2018).

[85] N. Craig, P. Draper, C. Kilic, and S. Thomas, Shedding light on diphoton resonances, Phys. Rev. D 93, 115023 (2016).

[86] ATLAS Collaboration, Search for resonances decaying to photon pairs in $139 \mathrm{fb}^{-1}$ of $p p$ collisions at $\sqrt{s}=13 \mathrm{TeV}$ with the ATLAS detector, .

[87] ATLAS Collaboration, Search for resonances in the 65 to $110 \mathrm{GeV}$ diphoton invariant mass range using $80 \mathrm{fb}^{-1}$ of $p p$ collisions collected at $\sqrt{s}=13 \mathrm{TeV}$ with the ATLAS detector (2018), https://cds.cern.ch/record/2628760.

[88] A. M. Sirunyan et al. (CMS Collaboration), Search for a standard model-like Higgs boson in the mass range between 70 and $110 \mathrm{GeV}$ in the diphoton final state in proton-proton collisions at $\sqrt{s}=8$ and $13 \mathrm{TeV}$, Phys. Lett. B 793, 320 (2019).

[89] G. Aad et al. (ATLAS Collaboration), Search for new phenomena in events with two opposite-charge leptons, jets and missing transverse momentum in $p p$ collisions at $\sqrt{s}=13 \mathrm{TeV}$ with the ATLAS detector, J. High Energy Phys. 04 (2021) 165.

[90] A. M. Sirunyan et al. (CMS Collaboration), Search for top squarks and dark matter particles in opposite-charge dilepton final states at $\sqrt{s}=13 \mathrm{TeV}$, Phys. Rev. D 97, 032009 (2018).

[91] K. Enqvist, J. Ignatius, K. Kajantie, and K. Rummukainen, Nucleation and bubble growth in a first order cosmological electroweak phase transition, Phys. Rev. D 45, 3415 (1992).
[92] A. D. Linde, Fate of the false vacuum at finite temperature: Theory and applications, Phys. Lett. 100B, 37 (1981).

[93] M. Quiros, Finite temperature field theory and phase transitions, arXiv:hep-ph/9901312.

[94] C. L. Wainwright, CosmoTransitions: Computing cosmological phase transition temperatures and bubble profiles with multiple fields, Comput. Phys. Commun. 183, 2006 (2012).

[95] J. M. Cline, K. Kainulainen, and D. Tucker-Smith, Electroweak baryogenesis from a dark sector, Phys. Rev. D 95, 115006 (2017).

[96] S. R. Coleman, The fate of the false vacuum. 1. Semiclassical theory, Phys. Rev. D 15, 2929 (1977).

[97] M. Kamionkowski, A. Kosowsky, and M. S. Turner, Gravitational radiation from first order phase transitions, Phys. Rev. D 49, 2837 (1994).

[98] J. R. Espinosa, T. Konstandin, J. M. No, and G. Servant, Energy budget of cosmological first-order phase transitions, J. Cosmol. Astropart. Phys. 06 (2010) 028.

[99] S. J. Huber and M. Sopena, An efficient approach to electroweak bubble velocities, arXiv:1302.1044.

[100] M. Hindmarsh, S. J. Huber, K. Rummukainen, and D. J. Weir, Shape of the acoustic gravitational wave power spectrum from a first order phase transition, Phys. Rev. D 96, 103520 (2017); , Erratum, Phys. Rev. D 101, 089902 (2020).

[101] A. Roper Pol, S. Mandal, A. Brandenburg, T. Kahniashvili, and A. Kosowsky, Numerical simulations of gravitational waves from early-universe turbulence, Phys. Rev. D 102, 083512 (2020).

[102] P. Amaro-Seoane et al. (LISA Collaboration), Laser interferometer space antenna, arXiv:1702.00786.

[103] V. Corbin and N. J. Cornish, Detecting the cosmic gravitational wave background with the big bang observer, Classical Quantum Gravity 23, 2435 (2006).

[104] E. Thrane and J.D. Romano, Sensitivity curves for searches for gravitational-wave backgrounds, Phys. Rev. D 88, 124032 (2013).

[105] M. Breitbach, J. Kopp, E. Madge, T. Opferkuch, and P. Schwaller, Dark, cold, and noisy: Constraining secluded hidden sectors with gravitational waves, J. Cosmol. Astropart. Phys. 07 (2019) 007.

[106] T. Alanne, T. Hugle, M. Platscher, and K. Schmitz, A fresh look at the gravitational-wave signal from cosmological phase transitions, J. High Energy Phys. 03 (2020) 004.

[107] K. Schmitz, New sensitivity curves for gravitational-wave signals from cosmological phase transitions, J. High Energy Phys. 01 (2021) 097.

[108] K. Schmitz, LISA sensitivity to gravitational waves from sound waves, Symmetry 12, 1477 (2020).

[109] E. Thrane and J. D. Romano, Sensitivity curves for searches for gravitational-wave backgrounds, Phys. Rev. D 88, 124032 (2013).

[110] G. D. Moore, Measuring the broken phase sphaleron rate nonperturbatively, Phys. Rev. D 59, 014503 (1998).

[111] K. Fuyuto and E. Senaha, Improved sphaleron decoupling condition and the Higgs coupling constants in the real singlet-extended Standard Model, Phys. Rev. D 90, 015015 (2014). 
[112] L. Fromme and S. J. Huber, Top transport in electroweak baryogenesis, J. High Energy Phys. 03 (2007) 049.

[113] M. D’Onofrio, K. Rummukainen, and A. Tranberg, Sphaleron Rate in the Minimal Standard Model, Phys. Rev. Lett. 113, 141602 (2014).

[114] J. M. Cline, K. Kainulainen, and M. Trott, Electroweak baryogenesis in two Higgs doublet models and B meson anomalies, J. High Energy Phys. 11 (2011) 089.

[115] A. Riotto, Towards a nonequilibrium quantum field theory approach to electroweak baryogenesis, Phys. Rev. D 53, 5834 (1996).

[116] A. Riotto, Supersymmetric electroweak baryogenesis, nonequilibrium field theory and quantum Boltzmann equations, Nucl. Phys. B518, 339 (1998).

[117] M. Joyce, T. Prokopec, and N. Turok, Nonlocal electroweak baryogenesis. Part 2: The Classical regime, Phys. Rev. D 53, 2958 (1996).

[118] J. M. Cline, M. Joyce, and K. Kainulainen, Supersymmetric electroweak baryogenesis, J. High Energy Phys. 07 (2000) 018.

[119] K. Kainulainen, T. Prokopec, M. G. Schmidt, and S. Weinstock, First principle derivation of semiclassical force for electroweak baryogenesis, J. High Energy Phys. 06 (2001) 031.

[120] K. Kainulainen, T. Prokopec, M. G. Schmidt, and S. Weinstock, Semiclassical force for electroweak baryogenesis: Three-dimensional derivation, Phys. Rev. D 66, 043502 (2002).

[121] T. Prokopec, M. G. Schmidt, and S. Weinstock, Transport equations for chiral fermions to order $\mathrm{h}$ bar and electroweak baryogenesis. Part 1, Ann. Phys. (Amsterdam) 314, 208 (2004).

[122] T. Prokopec, M. G. Schmidt, and S. Weinstock, Transport equations for chiral fermions to order h-bar and electroweak baryogenesis. Part II, Ann. Phys. (Amsterdam) 314, 267 (2004).

[123] H. Jukkala, K. Kainulainen, and O. Koskivaara, Quantum transport and the phase space structure of the Wightman functions, J. High Energy Phys. 01 (2020) 012.

[124] N. Aghanim et al. (Planck Collaboration), Planck 2018 results. VI. Cosmological parameters, Astron. Astrophys. 641, A6 (2020).

[125] T. Prokopec, J. Rezacek, and B. Świeżewska, Gravitational waves from conformal symmetry breaking, J. Cosmol. Astropart. Phys. 02 (2019) 009.
[126] C. Marzo, L. Marzola, and V. Vaskonen, Phase transition and vacuum stability in the classically conformal B-L model, Eur. Phys. J. C 79, 601 (2019).

[127] P. S. B. Dev, F. Ferrer, Y. Zhang, and Y. Zhang, Gravitational waves from first-order phase transition in a simple axion-like particle model, J. Cosmol. Astropart. Phys. 11 (2019) 006.

[128] Z. Kang, P. Ko, and T. Matsui, Strong first order EWPT \& strong gravitational waves in $\mathrm{Z}_{3}$-symmetric singlet scalar extension, J. High Energy Phys. 02 (2018) 115.

[129] A. Alves, T. Ghosh, H.-K. Guo, K. Sinha, and D. Vagie, Collider and gravitational wave complementarity in exploring the singlet extension of the standard model, J. High Energy Phys. 04 (2019) 052.

[130] X. Wang, F. P. Huang, and X. Zhang, Bubble wall velocity beyond leading-log approximation in electroweak phase transition, arXiv:2011.12903.

[131] J. Berges, S. Borsanyi, U. Reinosa, and J. Serreau, Nonperturbative renormalization for 2PI effective action techniques, Ann. Phys. (Amsterdm) 320, 344 (2005).

[132] A. Pilaftsis and D. Teresi, Exact RG invariance and symmetry improved 2PI effective potential, Nucl. Phys. B920, 298 (2017).

[133] T. Brauner, T. V. I. Tenkanen, A. Tranberg, A. Vuorinen, and D. J. Weir, Dimensional reduction of the Standard Model coupled to a new singlet scalar field, J. High Energy Phys. 03 (2017) 007.

[134] K. Kainulainen, V. Keus, L. Niemi, K. Rummukainen, T. V. I. Tenkanen, and V. Vaskonen, On the validity of perturbative studies of the electroweak phase transition in the Two Higgs Doublet model, J. High Energy Phys. 06 (2019) 075.

[135] D. Croon, O. Gould, P. Schicho, T. V. I. Tenkanen, and G. White, Theoretical uncertainties for cosmological firstorder phase transitions, J. High Energy Phys. 04 (2021) 055 .

[136] D. Cutting, M. Hindmarsh, and D. J. Weir, Vorticity, Kinetic Energy, and Suppressed Gravitational Wave Production in Strong First Order Phase Transitions, Phys. Rev. Lett. 125, 021302 (2020).

[137] D. Cutting, E. G. Escartin, M. Hindmarsh, and D. J. Weir, Gravitational waves from vacuum first order phase transitions II: From thin to thick walls, Phys. Rev. D 103, 023531 (2021). 